\title{
Dark energy perturbations and cosmic coincidence
}

\author{
Javier Grande, ${ }^{*}$ Ana Pelinson, ${ }^{\dagger}$ and Joan Solà \\ High Energy Physics Group, Departament d'ECM, and Institut de Ciències del Cosmos, Universitat de Barcelona, \\ Avinguda Diagonal 647, E-08028 Barcelona, Catalonia, Spain
}

(Received 6 December 2008; published 17 February 2009)

\begin{abstract}
While there is plentiful evidence in all fronts of experimental cosmology for the existence of a nonvanishing dark energy (DE) density $\rho_{D}$ in the Universe, we are still far away from having a fundamental understanding of its ultimate nature and of its current value, not even of the puzzling fact that $\rho_{D}$ is so close to the matter energy density $\rho_{M}$ at the present time (i.e. the so-called "cosmic coincidence" problem). The resolution of some of these cosmic conundrums suggests that the DE must have some (mild) dynamical behavior at the present time. In this paper, we examine some general properties of the simultaneous set of matter and DE perturbations $\left(\delta \rho_{M}, \delta \rho_{D}\right)$ for a multicomponent DE fluid. Next we put these properties to the test within the context of a nontrivial model of dynamical DE (the $\Lambda$ XCDM model) which has been previously studied in the literature. By requiring that the coupled system of perturbation equations for $\delta \rho_{M}$ and $\delta \rho_{D}$ has a smooth solution throughout the entire cosmological evolution, that the matter power spectrum is consistent with the data on structure formation, and that the "coincidence ratio" $r=\rho_{D} / \rho_{M}$ stays bounded and not unnaturally high, we are able to determine a well-defined region of the parameter space where the model can solve the cosmic coincidence problem in full compatibility with all known cosmological data.
\end{abstract}

DOI: 10.1103/PhysRevD.79.043006

\section{INTRODUCTION}

Undoubtedly the most prominent accomplishment of modern cosmology to date has been to provide strong indirect support for the existence of both dark matter (DM) and dark energy (DE) from independent data sets derived from the observation of distant supernovae [1], the anisotropies of the cosmic microwave background (CMB) [2], the lensing effects on the propagation of light through weak gravitational fields [3], and the inventory of cosmic matter from the large-scale structures (LSS) of the Universe [4,5]. But, in spite of these outstanding achievements, modern cosmology still fails to understand the ultimate physical nature of the components that build up the mysterious dark side of the Universe, most conspicuously the DE component of which the first significant experimental evidence was reported 10 years ago from supernovae observations. The current estimates of the DE energy density yield $\rho_{D}^{\exp } \simeq\left(2.4 \times 10^{-3} \mathrm{eV}\right)^{4}$ and it is believed that it constitutes roughly $70 \%$ of the total energy density budget for an essentially flat Universe. The big question now is: what is it from the point of view of fundamental physics? One possibility is that it is the ground state energy density associated to the quantum field theory (QFT) vacuum and, in this case, it is traditional to associate $\rho_{D}$ to $\rho_{\Lambda}=\Lambda / 8 \pi G$, where $\Lambda$ is the cosmological constant (CC) term in Einstein's equations. The problem, however, is that the typical value of the (renormalized) vacuum energy in all known realistic

\footnotetext{
*jgrande@ecm.ub.es †apelinson@ecm.ub.es

†sola@ifae.es
}

PACS numbers: 95.36.+x, 04.62.+v, 11.10.Hi

QFT's is much bigger than the experimental value. For example, the energy density associated to the Higgs potential of the standard model (SM) of electroweak interactions is more than 50 orders of magnitude larger than the measured value of $\rho_{D}$.

Another generic proposal (with many ramifications) is the possibility that the DE stands for the current value of the energy density of some slowly evolving, homogeneous, and isotropic scalar field (or a collection of them). Scalar fields appeared first as dynamical adjustment mechanisms for the CC [6,7] and later gave rise to the notion of quintessence [8]. While this idea has its own merits (especially concerning the dynamical character that confers to the DE) it has also its own drawbacks. The most obvious one (often completely ignored) is that the vacuum energy of the SM is still there and, therefore, the quintessence field just adds up more trouble to the whole fine-tuning $\mathrm{CC}$ problem $[9,10]$ !

Next-to-leading is the "cosmological coincidence problem," or the problem of understanding why the presently measured value of the DE is so close to the matter density. One expects that this problem can be alleviated by assuming that $\rho_{D}$ is actually a dynamical quantity. While quintessence is the traditionally explored option, in this paper we entertain the possibility that such dynamics could be the result of the so-called cosmological "constants" (like $\Lambda, G, \ldots$ ) being actually variable. It has been proven in [11] that this possibility can perfectly mimic quintessence. It means that we stay with the $\Lambda$ parameter and make it "running," for example, through quantum effects [1214]. ${ }^{1}$ However, in [17] it was shown that, in order to have

\footnotetext{
${ }^{1}$ For a general discussion, see $[15,16]$.
} 
an impact on the coincidence problem, the total DE in this context should be conceived as a composite fluid made out of a running $\Lambda$ and another entity $X$, with some effective equation of state (EOS) parameter $\omega_{X}$, such that the total DE density and pressure read $\rho_{D}=\rho_{\Lambda}+\rho_{X}$ and $p_{D}=$ $-\rho_{\Lambda}+\omega_{X} \rho_{X}$, respectively. We call this system the $\Lambda \mathrm{XCDM}$ model [17]. Let us emphasize that $X$ (called "the cosmon") is not necessarily a fundamental entity; in particular, it need not be an elementary scalar field. As remarked in [17], $X$ could represent the effective behavior of higher order terms in the effective action (including nonlocal ones). This is conceivable, since the Bianchi identity enforces a relation between all dynamical components that enter the effective structure of the energymomentum tensor in Einstein's equations, in particular, between the evolving $\Lambda$ and other terms that could emerge after we embed general relativity in a more general framework $[18,19]$. Therefore, at this level, we do not impose a microscopic description for $X$ and in this way the treatment becomes more general. ${ }^{2}$ The only condition defining $X$ is the DE conservation law, namely, we assume that $\rho_{D}=$ $\rho_{\Lambda}+\rho_{X}$ is the covariantly self-conserved total DE density.

In this paper, we analyze the combined dynamics of DE and matter density perturbations for such conserved DE density $\rho_{D}$. The present study goes beyond the approximate treatment presented in [23], where we neglected the DE perturbations and estimated the matter perturbations of the $\Lambda$ XCDM model using an effective (variable) EOS $w_{e}$ for the composite fluid $\left(\rho_{\Lambda}, \rho_{X}\right)$. The main result was that a sizeable portion of parameter space was still compatible with a possible solution of the cosmic coincidence problem. The "effective approach" that we employed in [23] was based on three essential ingredients: (i) the use of the effective EOS representation of cosmologies with variable cosmological parameters [11]; (ii) the calculation of the growth of matter density fluctuations using the effective EOS of the DE [24]; and (iii) the application of the, socalled, "F test" to compare the model with the LSS data, i.e. the condition that the linear bias parameter, $b^{2}(z)=$ $P_{G G} / P_{M M}$, does not deviate from the $\Lambda \mathrm{CDM}$ value by more than $10 \%$ at $z=0$, where $P_{M M} \propto\left(\delta \rho_{M} / \rho_{M}\right)^{2}$ is the matter power spectrum and $P_{G G}$ is the galaxy fluctuation power spectrum $[4,5]$ - see $[23,25]$ for details. This three-step methodology turned out to be an efficient streamlined strategy to further constrain the region of the original parameter space [17]. However, a full-fledged analysis of the system of cosmological perturbations in which the DE and matter fluctuations are coupled in a dynamical way remained to be performed. This kind of analysis is presented here.

\footnotetext{
${ }^{2}$ See e.g. [20-22] for recent constraints on $\Lambda C D M, X C D M$, and quintessencelike models. The margin for the energy densities and EOS parameter $\omega_{X}$ is still quite high. In the $\Lambda$ XCDM case, the fact that $\Lambda$ is running provides an even wider range of phenomenological possibilities.
}

The structure of the paper is as follows. In the next section, we outline the meaning of the cosmic coincidence problem within the general setting of the cosmological constant problem. In Sec. III, the basic equations for cosmological perturbations of a multicomponent fluid in the linear regime are introduced. In Sec. IV, we describe the general framework for addressing cosmological perturbations of a composite DE fluid with an effective EOS. In Sec. V, we describe some generic features of the cosmological perturbations for the dark energy component. The particular setup of the $\Lambda$ XCDM model is focused in Sec. VI. In Secs. VII and VIII, we put the $\Lambda$ XCDM model to the stringent test of cosmological perturbations and show that the corresponding region of parameter space becomes further reduced. Most important, in this region the model is compatible with all known observational data and, therefore, the $\Lambda \mathrm{XCDM}$ proposal can be finally presented as a robust candidate model for solving the cosmic coincidence problem. In Sec. IX, we offer a deeper insight into the correlation of matter and DE perturbations. In the last section, we present the final discussion and deliver our conclusions.

\section{THE COINCIDENCE PROBLEM AS A PART OF THE BIG CC PROBLEM}

The cosmic coincidence problem is a riddle, wrapped in the polyhedric mystery of the cosmological constant problem $[9,10]$, which has many faces. Indeed, we should clearly distinguish between the two main aspects which are hidden in the $\mathrm{CC}$ problem. In the first place, we have the "old CC problem" (the ugliest face of the CC conundrum), i.e. the formidable task of trying to explain the relatively small (for particle physics standards) measured value of $\rho_{\Lambda}$ or, more generally, of the DE density [1], roughly $\rho_{D}^{\exp } \sim 10^{-47} \mathrm{GeV}^{4}$, after the many phase transitions that our Universe has undergone since the very early times, in particular, the electroweak Higgs phase transition associated to the standard model of particle physics, whose natural value is in the ballpark of $\rho_{\mathrm{EW}} \sim G_{F}^{-2} \sim 10^{9} \mathrm{GeV}^{4}$ ( $G_{F}$ being Fermi's constant). The discrepancy $\rho_{\text {EW }} / \rho_{D}^{\exp }$, which amounts to some 56 orders of magnitude, is the biggest enigma of fundamental physics ever. ${ }^{3}$ Apart from the induced CC contribution from phase transitions, we have the pure vacuum-to-vacuum quantum effects. Since the (renormalized) zero point energy of a free particle of mass $m$ contributes $\sim m^{4}$ to the vacuum energy density $[12,13]$, it turns out that even a free electron contributes an amount more than 30 orders of magnitude larger than the aforementioned experimental value of $\rho_{D}$. Only light neutrinos $m_{\nu} \sim 10^{-3} \mathrm{eV}$, or scalar particles of similar mass, could contribute just the right amount, namely, if these

\footnotetext{
${ }^{3}$ See e.g. $[13,15,16]$ for a summarized presentation with specific insights closely related to the present approach.
} 
particles would be the sole active degrees of freedom in our present cold Universe (see [12]).

On the other hand, the cosmic coincidence problem [26] is that second ("minor") aspect of the CC problem addressing the specific question: "why just now?" i.e. why do we find ourselves in an epoch $t=t_{0}$ where the DE density is similar to the matter density, $\rho_{D}\left(t_{0}\right) \simeq \rho_{M}\left(t_{0}\right)$ ? In view of the rapidly decreasing value of $\rho_{M}(a) \sim 1 / a^{3}$, where $a=a(t)$ is the scale factor, it is quite puzzling to observe that its current value is precisely of the same order of magnitude as the vacuum energy or, in general, the dark energy density $\rho_{D}$. It is convenient to define the "cosmic coincidence ratio"

$$
r(a)=\frac{\rho_{D}(a)}{\rho_{M}(a)}=\frac{\Omega_{D}(a)}{\Omega_{M}(a)},
$$

where $\left(\Omega_{D}(a), \Omega_{M}(a)\right)$ are the corresponding densities normalized with respect to the current critical density $\rho_{c}^{0} \equiv$ $3 H_{0}^{2} / 8 \pi G$. For $\Omega_{M}^{0} \simeq 0.3$ and $\Omega_{D}^{0} \simeq 0.7$, we have $r_{0} \simeq 2.3$, which is of $\mathcal{O}(1)$. However, in the standard cosmological $\Lambda$ CDM model, where $\Omega_{D}$ is constant and $\Omega_{M}(a \rightarrow \infty) \rightarrow$ 0 , the ratio $r$ grows unboundedly with the expansion of the Universe. So the fact that $r_{0}=\mathcal{O}(1)$ is regarded as a puzzle because it suggests that $t=t_{0}$ is a very special epoch of our Universe. One could also consider the inverse ratio $r^{-1}=$ $\rho_{M}(a) / \rho_{D}(a)$, which goes to zero with the expansion. The coincidence problem can be equivalently formulated either by asking why $r$ is not very large now or why $r^{-1}$ is not very small. Solving the coincidence problem would be to find either (1) a concrete explanation for $r$ and $r^{-1}$ being of order one at present within the standard cosmological model, or (2) a modified cosmological model (compatible with all known cosmological data) insuring that these ratios do not undergo a substantial change, say by more than 1 order of magnitude or so, for a very long period of the cosmic history that includes our time.

In a very simplified way, let us summarize some of the possible avenues that have been entertained to cope with the coincidence puzzle:

(i) Quintessence and the like [8,27-30]. One postulates the existence of a set of cosmological scalar fields $\phi_{i}$ essentially unrelated to the rest of the particle physics world. The DE produced by these fields has an effective EOS parameter $\omega_{D} \gtrsim-1$ which causes $\rho_{D}$ to decrease always with the expansion (i.e. $d \rho_{D} / d a<0$ ), but at a pace slower (on average) than that of the background matter. Thus, it finally catches up with it and $\rho_{D}$ emerges to the surface, i.e. the condition $\rho_{D}>\rho_{M}$ eventually holds (presumably near our time). In this framework, there is the possibility of self-adjusting and tracker solutions $[8,27]$, where the DE keeps track of the matter behavior and ultimately dominates the Universe. It requires to take some special forms of the potential, and in some cases the Lagrangian involves nonca- nonical kinetic terms. For example, in the simple case of a single scalar field and the exponential potential $V(\phi) \sim \exp \left(-\lambda \phi / M_{P}\right)$ one finds that the coincidence ratio becomes fixed at the value

$$
r=\frac{3\left(1+\omega_{m}\right)}{\lambda^{2}-3\left(1+\omega_{m}\right)},
$$

where $\omega_{m}$ is the EOS of the background matter (i.e. 0 or $1 / 3$, depending on whether cold or relativistic matter dominates, respectively). So, at the present time, $r=3 /\left(\lambda^{2}-3\right)$, and by the appropriate choice of $\lambda$ one can match the current experimental value. But, of course, the choice of the potential was rather peculiar and the field $\phi$ itself is completely ad hoc. Moreover, it has a mass $m_{\phi}=\sqrt{V^{\prime \prime}(\phi)} \sim H \sim$ $10^{-33} \mathrm{eV}$ (as it follows from a self-consistent solution of Einstein's equations); such mass scale is 30 orders of magnitude below the mass scale associated to the DE, which is $\rho_{D}^{1 / 4} \sim 10^{-3} \mathrm{eV}$. In this sense, it looks a bit unnatural to aim at solving the CC problem by introducing a field whose extremely tiny mass creates another cosmological puzzle.

On the other hand, within the context of interactive quintessence models [28] (whose main leitmotif is precisely trying to cure the coincidence problem), the coupling of $\phi_{i}$ and the matter components makes an allowance for energy exchange between the two kinds of fields, and as a result the ratio (1) can be constant or slowly variable, whereas in other implementations one can achieve an oscillatory tracking behavior of $r$, although the construction is essentially ad hoc [29]. Another generalization leads to $k$-essence models [30] (characterized by noncanonical kinetic terms), where fine-tuning problems in the tracking can be disposed of but the dominant background component can be tracked only up to matterradiation equality and is lost immediately afterwards (as the DE is immediately prompted into a CC-like behavior). In one way or another, however, all variants of quintessence suffer from several drawbacks, and, in particular, from the following generic one: they assume (somehow implicitly) that the remaining fields of the particle physics spectrum (i.e. those which were already there from the very beginning) have nothing to do with the $\mathrm{CC}$ problem. As a result of such a bold assumption, the (likely real) vacuum problem of the conventional fields in QFT is merely traded for the (likely fictitious) vacuum problem of quintessence, which is no less acute because no real explanation is provided for the smallness of the current $\rho_{D}$ value versus $m^{4}$ (where $m$ is any typical mass scale in particle physics). Hence we are back to the same kind of CC problem we started with.

(ii) Phantom energy [31]. It is motivated by the fact that, observationally speaking, the effective EOS of the 
DE cannot be excluded to satisfy $\omega_{D} \lesssim-1$ near our present time. As indicated above, many quintessencelike models in reality are hybrid constructions containing a mixture of fields with a phantom component. The reason is that one wants to give allowance for a "CC-crossing" $\omega_{D}=-1$ near our time. While phantom energy shares with quintessence the use of scalar fields $\phi_{i}$, here the DE produced by these fields is always increasing with the expansion, $d \rho_{D} / d a>0$, even after the relation $\rho_{D}>\rho_{M}$ is fulfilled. The consequence of this ever growing behavior of the dark component is that one ends up with a superaccelerated expansion of the Universe that triggers an eventual disruption of all forms of matter (the so-called "big rip"). When computing the fraction of the lifetime of the Universe where the ratio (1) stays within given bounds before the "doomsday," one finds that it can be sizeable.

(iii) Nonlocal theories. There is some renewed interest in these kinds of theories in which the emphasis is placed on the existence of possible nonlocal structures in the effective action [32]. It has recently been emphasized in [16] that the dynamical evolution of the vacuum energy should come from a resummation of terms in the effective action leading to nonlocal contributions of the form $R \mathcal{F}\left(G_{0} R\right)$, for some unknown function $\mathcal{F}$ of dimension 2 , where $G_{0}$ is the massless Green's function $\left(G_{0} \sim 1 / \square\right)$. The canonical possibility would be $\mathcal{F}=M^{2} G_{0} R$, where $M$ is a parameter with dimension of mass. This situation leads to an effective evolution of the CC of the form $\Delta \rho_{\Lambda} \sim$ $M^{2} H^{2}$ during the matter-dominated epoch, whereas in the radiation era the effective $\mathrm{CC}$ would approximately be zero (because $R \sim T_{\mu}^{\mu} \simeq 0$ for relativistic matter, see (12) and (13) below). As a result, the coincidence puzzle could somehow be understood from the fact that the CC may start to be preponderant at some point once the onset of the matterdominated epoch is left behind.

(iv) Of course many other ideas have been explored. For instance, one may introduce special fluids with very peculiar EOS, such as the Chaplygin gas [33], which behaves as pressureless matter at early times $\left(\omega_{D} \simeq 0\right)$ and as vacuum energy at present $\left(\omega_{D} \simeq\right.$ $-1)$. Although there is some connection with braneworld cosmology, this proposal suffers from the same problem as quintessence in that it supersedes the vacuum state of traditional fields by the new vacuum of that peculiar fluid. Finally, let us mention the anthropic models, which fall in a quite different category, in the sense that one does not look for a solution of the coincidence problem exclusively from first principles of QFT or string theory, but rather through the interplay of the "human factor." Basically, one ties the value of the ratio (1) to the time when the conditions arise for the development of intelligent life in the Universe, in particular, of cosmologists making observations of the cosmos. This variant has also a long story, but we shall refrain from entering the details, see e.g. [9,34].

\section{COSMOLOGICAL PERTURBATIONS FOR A MULTICOMPONENT FLUID IN THE LINEAR REGIME}

In the remainder of the paper we concentrate on studying some general properties of the cosmological perturbations, both of matter and DE, and the implications they may have on the coincidence problem within models characterized by having running vacuum energy and other DE components. According to cosmological perturbation theory, all energy density components, including the dark energy, should fluctuate and contribute to the growth of the large-scale cosmological structures. In this section, we discuss the general framework of linear density perturbations in models composed of a multicomponent DE fluid besides the canonical matter.

In the following we use the standard metric perturbation approach [35] and consider simultaneous density and pressure perturbations

$$
\rho_{N} \rightarrow \rho_{N}+\delta \rho_{N}, \quad p_{N} \rightarrow p_{N}+\delta p_{N},
$$

for all the components $(N=1,2, \ldots)$ of the fluid, including matter and all possible contributions from the multicomponent DE part. At the same time, we have metric and velocity perturbations for each component:

$$
g_{\mu \nu}^{B} \rightarrow g_{\mu \nu}=g_{\mu \nu}^{B}+\delta g_{\mu \nu}, \quad U_{N}^{\mu} \rightarrow U_{N}^{\mu}+\delta U_{N}^{\mu},
$$

where $g_{\mu \nu}^{B}$ is the background metric. The four-vector velocity $U_{N}^{\mu}$ in the comoving coordinates has the following components and perturbations $\left(U_{N}^{0}, U_{N}^{i}\right)=(1,0) \rightarrow$ $\delta U_{N}^{\mu}=\left(0, v_{N}^{i}\right)$, where $v_{N}^{i}$ is the three-velocity of the $N$ th component of the fluid in the chosen coordinate system. As a background space-time, we assume the homogeneous and isotropic Friedmann-Lemaître-Robertson-Walker (FLRW) metric with flat space section, hence $g_{\mu \nu}^{B}=$ $\operatorname{diag}\left\{1,-a^{2}(t) \delta_{i j}\right\}$, where $a$ is the scale factor.

In order to derive the set of perturbed equations, let us first introduce Einstein's equations:

$$
R_{\mu \nu}-\frac{1}{2} R g_{\mu \nu}=8 \pi G T_{\mu \nu},
$$

where $G$ is the Newton constant and $T_{\mu \nu}$ is the total energy-momentum tensor of matter and dark energy. Both the background and perturbed metric are assumed to satisfy these equations. The total energy-momentum tensor of the system is assumed to be the sum of the perfect fluid form for each component: 


$$
T_{\mu \nu}=\sum_{N} T_{\mu \nu}^{N}=\sum_{N}\left[-p_{N} g_{\mu \nu}+\left(\rho_{N}+p_{N}\right) U_{\mu}^{N} U_{\nu}^{N}\right] .
$$

The components of $T_{\nu}^{\mu}$ are the following:

$$
\begin{gathered}
T_{0}^{0}=\sum_{N} \rho_{N} \equiv \rho_{T}, \\
T_{j}^{i}=-\sum_{N} p_{N} \delta_{j}^{i} \equiv-p_{T} \delta_{j}^{i},
\end{gathered}
$$

where $\rho_{T}$ and $p_{T}$ are the total energy density and pressure, respectively.

Perturbations on the metric and on the energymomentum tensor are uniquely defined for a given perturbed space-time, provided we make a gauge choice. The latter means that we choose a specific coordinate system; in this way, four out of the 10 components $\delta g_{\mu \nu} \equiv h_{\mu \nu}$ of the metric perturbation can be fixed at will. Here we have adopted the synchronous gauge, widely used in the literature, in which the four preassigned values of the metric perturbations are $h_{0 i}=0$ and $h_{00}=0$. Setting $h_{\mu \nu}=$ $-a^{2} \chi_{\mu \nu}$, in this gauge the perturbed, spatially flat, FLRW metric takes on the form

$$
\begin{aligned}
d s^{2} & =g_{\mu \nu} d x^{\mu} d x^{\nu}=d t^{2}-a^{2}(t)\left(\delta_{i j}+\chi_{i j}\right) d x^{i} d x^{j} \\
& =a^{2}(\eta)\left[d \eta^{2}-\left(\delta_{i j}+\chi_{i j}\right) d x^{i} d x^{j}\right],
\end{aligned}
$$

where in the last equality we have expressed the result also in terms of the conformal time $\eta$, defined through $d \eta=$ $d t / a$. We may compare (9) with the most general perturbation of the spatially flat FLRW metric,

$$
\begin{aligned}
d s^{2}= & a^{2}(\eta)\left[(1+2 \psi) d \eta^{2}-\omega_{i} d \eta d x^{i}\right. \\
& \left.-\left((1-2 \phi) \delta_{i j}+\chi_{i j}\right) d x^{i} d x^{j}\right],
\end{aligned}
$$

consisting of the 10 degrees of freedom associated to the two scalar functions $\psi, \phi$, the three components of the vector function $\omega_{i}(i=1,2,3)$, and the five components of the traceless $\chi_{i j}$. Clearly, the synchronous gauge (9) is obtained by setting $\psi=0, \omega_{i}=0$ and absorbing the function $\phi$ into the trace of $\chi_{i j}$. In this way, $\chi_{i j}$ in (9) contributes six degrees of freedom. As we will see in a moment, in practice only the nonvanishing trace of the metric disturbance will be necessary to perform the analysis of cosmic perturbations in this gauge. To within first order of perturbation theory, such a trace is given by

$$
h \equiv g^{\mu \nu} h_{\mu \nu}=g^{i j} h_{i j}=-\frac{h_{i i}}{a^{2}}=\chi_{i i},
$$

where $g^{i j}$ is the inverse of $g_{i j}=-a^{2}(t)\left(\delta_{i j}+\chi_{i j}\right)$, and it is understood that the repeated Latin indices are summed over $1,2,3$.

For the physical interpretation, notice that the synchronous gauge is associated to a coordinate system in which the cosmic time coordinate is comoving with the fluid particles $\left(g_{00}=1\right.$, i.e. $\left.\psi=0\right)$, which is the reason for its name and also explains why this gauge does not have an obvious Newtonian limit. In fact, this gauge choice is generally appropriate for the study of fluctuations whose wavelength is larger than the Hubble radius $\left(\lambda \gg d_{H} \equiv\right.$ $H^{-1}$ ). Actually, any mode satisfies this condition at sufficiently early epochs, and in this regime the effects of the space-time curvature are unavoidable.

Next we wish to compute the perturbations in the synchronous gauge. To start with, it is convenient to rewrite Einstein's equations (5) as follows:

$$
R_{\mu \nu}=8 \pi G S_{\mu \nu}, \quad S_{\mu \nu} \equiv T_{\mu \nu}-\frac{1}{2} g_{\mu \nu} T,
$$

where $T=T_{\mu}^{\mu}$ is the trace of (6), hence

$$
T=\sum_{N}\left(\rho_{N}-3 p_{N}\right)
$$

For the calculation of the perturbations, we can use any of the components of Einstein's equations. However, since we are going to use the conservation law $\nabla_{\mu} T_{\nu}^{\mu}=0$ to derive additional fluctuation equations, it is convenient to perturb the (00)-component of (12) because the other components are well known not to be independent of the conservation law. Thus, using (6), (12), and (13) we obtain

$$
\begin{aligned}
S_{00} & =T_{00}-\frac{1}{2} T=\frac{1}{2} \sum_{N}\left(\rho_{N}+3 p_{N}\right) \Rightarrow \delta S_{00} \\
& =\frac{1}{2} \sum_{N}\left(\delta \rho_{N}+3 \delta p_{N}\right) .
\end{aligned}
$$

On the other hand, a straightforward calculation shows that the perturbation of the (00)-component of the Ricci tensor can be written as follows:

$$
\delta R_{00}=-\frac{1}{2} \frac{\partial^{2} h}{\partial t^{2}}-H \frac{\partial h}{\partial t}=\frac{1}{2} \hat{h}+H \hat{h},
$$

where we have used (11) and defined the "hat variable"

$$
\hat{h} \equiv \frac{\partial}{\partial t}\left(\frac{h_{i i}}{a^{2}}\right)=-\stackrel{\circ}{h} .
$$

The overhead circle $(\circ)$ indicates partial differentiation with respect to the cosmic time (i.e. $f \equiv \partial f / \partial t$, for any $f$ ), in order to distinguish it from other differentiations to be used later on. Therefore, $H=\stackrel{\circ}{a} / a$ is the ordinary expansion rate in the cosmic time $t$.

Since the fluctuations $\delta S_{00}$ and $\delta R_{00}$ from (14) and (15) are constrained by (12), we find

$$
\stackrel{\circ}{h}+2 H \hat{h}=8 \pi G \sum_{N}\left(\delta \rho_{N}+3 \delta p_{N}\right) .
$$

If we substitute (16) in the previous expression, a secondorder differential equation in the original variable (11) ensues. In terms of the conformal time $\eta$, it can be written as follows: 


$$
\ddot{h}+\mathcal{H} \dot{h}=-8 \pi G a^{2} \sum_{N}\left(\delta \rho_{N}+3 \delta p_{N}\right),
$$

where the dot () indicates differentiation with respect to $\eta$ (i.e. $\dot{f} \equiv d f / d \eta$ ) and $\mathcal{H} \equiv \dot{a} / a=H a$ is the expansion rate in the conformal time.

The Friedmann equation can be written in terms of the normalized densities as

$$
H^{2}=\frac{8 \pi G}{3} \rho_{T}=H_{0}^{2} \sum_{N} \Omega_{N},
$$

where $H_{0}$ is the present value of the Hubble parameter and $\Omega_{N} \equiv \rho_{N} / \rho_{c}^{0}$ are the normalized densities with respect to the current critical density $\rho_{c}^{0} \equiv 3 H_{0}^{2} / 8 \pi G$.

The subsequent step is to perform perturbations on the conservation law $\nabla_{\mu} T^{\mu \nu}=0$, as this will provide the additional independent equations. Using (6), the previous law reads explicitly as follows:

$$
\begin{aligned}
& \sum_{N}\left\{\nabla_{\mu}\left(\rho_{N}+p_{N}\right) U_{N}^{\mu} U_{N}^{\nu}+\left(\rho_{N}+p_{N}\right)\right. \\
&\left.\quad \times\left[U_{N}^{\nu} \nabla_{\mu} U_{N}^{\mu}+U_{N}^{\mu} \nabla_{\mu} U_{N}^{\nu}\right]\right\}=g^{\mu \nu} \sum_{N} \nabla_{\mu} p_{N} .
\end{aligned}
$$

For any four-velocity vector, we have $U_{N}^{\mu} U_{\mu}^{N}=1$ and, therefore, we have the orthogonality relation $U_{\nu}^{N} \nabla_{\mu} U_{N}^{\nu}=$ 0 . In this way, by contracting Eq. (20) with $U_{\nu}^{N}$ we find the simpler result

$$
\sum_{N}\left[U_{N}^{\mu} \nabla_{\mu} \rho_{N}+\left(\rho_{N}+p_{N}\right) \nabla_{\mu} U_{N}^{\mu}\right]=0 .
$$

Let us emphasize that the sum $\sum_{N}$ in this equation need not run necessarily over all the terms of the cosmic fluid. It may hold for particular subsets of fluid components that are overall self-conserved. In particular, it could even hold for each component, if they would be individually conserved. In our case, it applies to the specific matter component and also, collectively, to the multicomponent DE part. It is straightforward to check that, in the FLRW metric, we have

$$
\nabla_{\mu} U_{N}^{\mu}=3 H \quad(N=1,2, \ldots) .
$$

Using this relation, it is immediate to see that, in the comoving frame, Eq. (21) boils down to

$$
\sum_{N}\left[\stackrel{\circ}{\rho}_{N}+3 H\left(\rho_{N}+p_{N}\right)\right]=0 .
$$

Moreover, perturbing (22) in the synchronous gauge, we find (using $\delta \Gamma_{\mu 0}^{\mu}=-\hat{h} / 2$ for the perturbed Christoffel symbol involved in the covariant derivative) the useful result

$$
\delta H=\frac{1}{3} \delta\left(\nabla_{\mu} U_{N}^{\mu}\right)=\frac{1}{3}\left(\theta_{N}-\frac{\hat{h}}{2}\right),
$$

where we have introduced the notation $\theta_{N} \equiv \nabla_{\mu}\left(\delta U_{N}^{\mu}\right)=$ $\nabla_{i}\left(\delta U_{N}^{i}\right)$ (with $\delta U_{N}^{0}=0$ ) for the covariant derivative of the perturbed three-velocity $\delta U_{N}^{i}=v_{N}^{i}$. Equipped with these formulas, the perturbed equation (23) immediately leads to

$\sum_{N}\left[\delta \stackrel{\circ}{\rho}_{N}+\left(\rho_{N}+p_{N}\right)\left(\theta_{N}-\frac{\hat{h}}{2}\right)+3 H\left(\delta \rho_{N}+\delta p_{N}\right)\right]=0$.

The previous result could have equivalently been obtained by setting $\nu=0$ in (20) and perturbing the corresponding equation. An independent relation can be obtained by setting $\nu=i$ in (20) within the comoving frame and carrying out the perturbation. Using the relevant Christoffel symbol $\Gamma_{0 j}^{i}=H \delta_{i j}$ and Eq. (22) we obtain, after some calculations,

$\sum_{N}\left\{\left(\rho_{N}+p_{N}\right) \delta \stackrel{\circ}{U}_{N}^{i}+\left[\stackrel{\circ}{\rho}_{N}+\stackrel{\circ}{p}_{N}+5 H\left(\rho_{N}+p_{N}\right)\right] \delta U_{N}^{i}\right\}$

$=\sum_{N} \nabla^{i} \delta p_{N}$

The final step is obtained by computing the divergence $\nabla_{i}$ on both sides of this equation. To within first order of perturbation theory, we obtain $\nabla_{i} \nabla^{i} \delta p_{N}=$ $g^{i k} \nabla_{i} \nabla_{k} \delta p_{N}=-\left(1 / a^{2}\right) \nabla^{2} \delta p_{N}$ for the action of $\nabla_{i}$ on the right-hand side (r.h.s.) of (26), whereas on its lefthand side (1.h.s.) we can use the variable $\theta_{N}$ previously defined. Moreover, we have $\nabla_{i} \delta \stackrel{\circ}{U}_{N}^{i}=\stackrel{\circ}{\theta}_{N}$ owing to the commutativity of coordinate differentiation and perturbation operations. In this way, the final outcome reads

$$
\begin{aligned}
& \sum_{N}\left\{\left(\rho_{N}+p_{N}\right) \stackrel{\circ}{\theta}_{N}+\left[\stackrel{\circ}{\rho}_{N}+\stackrel{\circ}{p}_{N}+5 H\left(\rho_{N}+p_{N}\right)\right] \theta_{N}\right\} \\
& =\frac{k^{2}}{a^{2}} \sum_{N} \delta p_{N},
\end{aligned}
$$

where it is furthermore understood that we have used the Fourier decomposition for all the perturbation variables:

$$
\delta f(t, \mathbf{x})=\int \frac{d^{3} k}{(2 \pi)^{3}} \delta f(t, k) e^{i \mathbf{k} \cdot \mathbf{x}},
$$

where $\delta f=\left(\hat{h}, \delta \rho_{N}, \delta p_{N}, \theta_{N}\right)$. In Fourier space, the perturbation variables are denoted with the same notation, but they are the Fourier transforms of the original ones, so their arguments are $t$ and $k$ because the space variable $\mathbf{x}$ has been traded for the wave number $k \equiv|\mathbf{k}|$. The latter will be measured in units of $h \mathrm{Mpc}^{-1}$, where $h \simeq 0.7$ is the reduced Hubble constant - not to be confused with the trace of the synchronous perturbed metric, Eq. (11). In these units, the linear regime corresponds to length scales $\ell \sim$ $k^{-1}$ with wave numbers $k<0.2 h \mathrm{Mpc}^{-1}$, i.e. $\ell>$ $5 h^{-1} \mathrm{Mpc}$. Notice that, if desired, one can easily rewrite the above perturbation equations (25) and (27) in conformal time simply by using $\stackrel{\circ}{f}=\dot{f} / a$ for any $f$. 
We have obtained three basic sets of perturbation equations (17), (25), and (27) for the four kinds of perturbation variables $\left(\hat{h}(t, k), \delta \rho_{N}(t, k), \delta p_{N}(t, k), \theta_{N}(t, k)\right)$. It is thus clear that the evolution of the cosmic perturbations can be completely specified only after we assume some relation of the pressure perturbation $\delta p_{N}$ and the density perturbation $\delta \rho_{N}$ for each fluid. If the perturbations are adiabatic, then that relation is simply

$$
\delta p_{N}=c_{a, N}^{2} \delta \rho_{N},
$$

where $c_{a, N}^{2}$ is the adiabatic speed of sound for each fluid, defined as

$$
c_{a, N}^{2} \equiv \frac{\dot{p}_{N}}{\dot{\rho}_{N}},
$$

where the dot differentiation here is with respect to whatever definition of time. Notice that if the various components would have an EOS of the form $p_{N}=w_{N} \rho_{N}$, with constant EOS parameter $w_{N}$, then $c_{a, N}^{2}=w_{N}$. However, even in this case the mixture has a variable effective EOS parameter, as we will see in the next section.

On the other hand, if the perturbations are nonadiabatic, there is an entropy contribution to the pressure perturbation [36]:

$$
p_{N} \Gamma_{N}=\delta p_{N}-c_{a, N}^{2} \delta \rho_{N},
$$

where $\Gamma_{N} \equiv\left(\delta p_{N}\right)_{\text {nonadiabatic }} / p_{N}$ is the intrinsic entropy perturbation of the $N$ th component, representing the displacement between hypersurfaces of uniform pressure and uniform energy density [37]. For covariantly conserved components, a gauge-invariant relationship between $\delta p_{N}$ and $\delta \rho_{N}$ for a general nonadiabatic stress is given by [3740]:

$$
\delta p_{N}=c_{s, N}^{2} \delta \rho_{N}+3 H a^{2}\left(\rho_{N}+p_{N}\right)\left(c_{s, N}^{2}-c_{a, N}^{2}\right) \frac{\theta_{N}}{k^{2}},
$$

where $c_{s, N}^{2}$ can be regarded as a rest frame speed of sound. We will refer to $c_{s, N}^{2}$ as the effective speed of sound, in the sense that we treat the cosmic fluid effectively as hydrodynamical matter. Since (32) is gauge invariant, the perturbed quantities in this expression can be computed, in particular, within the synchronous gauge. In this way, we can consistently substitute (32) in the Eqs. (17), (25), and (27) to eliminate the perturbation $\delta p_{N}$. This allows us, finally, to solve for the three basic sets of perturbations

$$
\left(\hat{h}(t, k), \delta \rho_{N}(t, k), \theta_{N}(t, k)\right) .
$$

\section{PERTURBATIONS FOR A COMPOSITE DE FLUID WITH A VARIABLE EFFECTIVE EQUATION OF STATE}

In this section, we apply the linear matter and dark energy density perturbations to a general class of models in which the DE fluid is a composite and covariantly selfconserved medium and matter is also canonically conserved. From Eq. (23), in the matter-dominated epoch $\left(p_{M}=0\right)$, the matter component $\rho_{M}$ satisfies

$$
\rho_{M}^{\prime}(a)+\frac{3}{a} \rho_{M}(a)=0 .
$$

Here we found it convenient to trade the differentiation with respect to the cosmic time ( $\circ$ ) for the differentiation with respect to the scale factor. The latter is denoted by a prime (i.e. $f^{\prime} \equiv d f / d a$ for any $f$; hence $f=a H f^{\prime}$ ). The scale factor is related to the cosmological redshift $z$ by $a(z) \equiv 1 /(1+z)$, where we define $a(0) \equiv a_{0}=1$ at the present time. It follows that the normalized matter density evolves as

$$
\Omega_{M}(a)=\Omega_{M}^{0} a^{-3},
$$

where $\Omega_{M}^{0}$ is the corresponding current value. Since the total DE is also globally conserved, from Eq. (23) we also obtain

$$
\rho_{D}^{\prime}+\frac{3}{a}\left(1+w_{e}\right) \rho_{D}=0
$$

where $w_{e}$ is the effective EOS parameter and $\rho_{D}=\rho_{1}+$ $\rho_{2}+\ldots$ is the total density of the multicomponent DE fluid. For a composite DE model, in which the DE is a mixture of fluids with individual EOS $p_{i}=\omega_{i} \rho_{i}(i=$ $1,2, \ldots, n)$, the effective EOS parameter is defined as

$$
w_{e}=\frac{p_{D}}{\rho_{D}}=\frac{\omega_{1} \rho_{1}+\omega_{2} \rho_{2}+\ldots}{\rho_{1}+\rho_{2}+\ldots} .
$$

The Hubble expansion in terms of the normalized densities, in the matter-dominated period, follows from (19)

$$
H^{2}=\frac{8 \pi G}{3} \rho_{T}=H_{0}^{2}\left(\Omega_{M}^{0} a^{-3}+\Omega_{D}(a)\right),
$$

where $\Omega_{D}$ is the normalized total DE density $\Omega_{D}(a) \equiv$ $\rho_{D}(a) / \rho_{c}^{0}$.

The nonadiabatic perturbed pressure (32) for the total DE component can be written in terms of the effective EOS as $^{4}$

$$
\delta p_{D}=c_{s}^{2} \delta \rho_{D}+3 H a^{2}\left(1+w_{e}\right) \rho_{D}\left(c_{s}^{2}-c_{a}^{2}\right) \frac{\theta_{D}}{k^{2}},
$$

where we have omitted for simplicity the subindex ' $D$ ' from the adiabatic and effective speeds of sound of the DE (i.e. $c_{a}^{2} \equiv c_{a, D}^{2}, c_{s}^{2} \equiv c_{s, D}^{2}$; this convention will be used throughout the text). The units are taken to be the light speed $c=1$ and $\hbar=1$ such that the Planck scale is defined by $M_{P}=G^{-1 / 2}=1.22 \times 10^{19} \mathrm{GeV}$. In these units, usu-

\footnotetext{
${ }^{4}$ In the particular case where the DE is a purely running $\Lambda$, one has to consider the perturbations $\delta \rho_{\Lambda}$, but since $\omega_{\Lambda}=-1$ it turns out that $\theta_{D}$ (here $\theta_{\Lambda}$ ) plays no role and hence it is enough to consider the relation $\delta p_{\Lambda}=-\delta \rho_{\Lambda}$, see Ref. [41] for details.
} 
ally $0 \leq c_{s}^{2} \leq 1$ for a general DE model. In this range, one can show that for constant EOS parameter there is small suppression on the DE fluctuation $\delta \rho_{D}$ as $c_{s}^{2}$ increases [37]. Near-zero (but not vanishing) sound speed today is possible in models like $k$-essence, for example, in which the EOS parameter is positive until the matter-dominated triggers a change to negative pressure; in this kind of model, it is even possible to have $c_{s}^{2}>1$, a regime for which the growth of the DE density perturbations is suppressed [42].

In a nonperfect fluid, spatial inhomogeneities in $T_{\mu \nu}$ imply shear viscosity in the fluid. In this case, a possible contribution to shear through a "viscosity parameter" $c_{\mathrm{vis}}^{2}$ should also be taken into account [43]. In principle, $c_{s}^{2}$ is an arbitrary parameter. Nevertheless, the limit where $\left(c_{s}^{2}, c_{\mathrm{vis}}^{2}\right) \rightarrow(1,0)$ corresponds exactly to a scalar field component with canonical kinetic term [44].

Using the total DE conservation law (36), we can write the total DE adiabatic sound speed (30) as

$$
c_{a}^{2} \equiv \frac{p_{D}^{\prime}}{\rho_{D}^{\prime}}=w_{e}-\frac{a}{3} \frac{w_{e}^{\prime}}{\left(1+w_{e}\right)} .
$$

The perturbed equations (25) and (27) for the (conserved) matter component (for which $p_{M}=\delta p_{M}=0$ ) can be written as differential equations in the scale factor:

$$
\begin{gathered}
\delta_{M}^{\prime}=-\frac{1}{a H}\left(\theta_{M}-\frac{\hat{h}}{2}\right), \\
\theta_{M}^{\prime}=-\frac{2}{a} \theta_{M},
\end{gathered}
$$

where $\delta_{M} \equiv \delta \rho_{M} / \rho_{M}$ is the relative matter fluctuation (density contrast). According to Eq. (42), the matter velocity gradient is decaying $\left(\theta_{M} \propto a^{-2}\right)$. Assuming the conventional initial condition $\theta_{M}^{0} \equiv \theta_{M}(a=1)=0$, we have $\theta_{M}(a)=0(\forall a)$. So, the perturbed matter set of coupled equations (41) and (42) yields the simple relation

$$
\hat{h}=2 a H \delta_{M}^{\prime}
$$

Let us also define the relative fluctuation of the DE component, $\delta_{D} \equiv \delta \rho_{D} / \rho_{D}$. Using the nonadiabatic perturbed pressure (39) and the DE conservation law (36), we can write the perturbed equations (25) and (27) for the selfconserved DE fluid in the following way:

$$
\begin{gathered}
\delta_{D}^{\prime}=-\frac{\left(1+w_{e}\right)}{a H}\left\{\left[1+\frac{9 a^{2} H^{2}\left(c_{s}^{2}-c_{a}^{2}\right)}{k^{2}}\right] \theta_{D}-\frac{\hat{h}}{2}\right\} \\
-\frac{3}{a}\left(c_{s}^{2}-w_{e}\right) \delta_{D}, \\
\theta_{D}^{\prime}=-\frac{1}{a}\left(2-3 c_{s}^{2}\right) \theta_{D}+\frac{k^{2}}{a^{3} H} \frac{c_{s}^{2} \delta_{D}}{\left(1+w_{e}\right)},
\end{gathered}
$$

where in the last equation we have used (40) to eliminate $c_{a}^{2}$. Moreover, from (45) one can see that a negligible DE sound speed $\left(c_{s}^{2} \approx 0\right)$ causes the velocity gradient to decay $\left(\theta_{D} \propto a^{-2}\right)$, as in the case of matter [Eq. (42)]. If we assume the conventional initial conditions $\theta_{M}^{0}=\theta_{D}^{0}=0$, we have $\theta_{M}=\theta_{D}=0(\forall a)$. In this case, the total DE fluid is comoving with the matter as long as the Universe and perturbations evolve, which is a very particular case. Actually, for this case, the $k$ (scale) dependence disappears from the equations. On the other hand, from Eq. (45) one can see that, if we neglect the DE perturbations, $\delta \rho_{D} \approx 0$, for a constant $c_{s}^{2}$ we obtain again $\theta_{M}=\theta_{D}=0$ and the scale independence.

However, $\delta \rho_{D}$ modifies the evolution of the metric fluctuations according to the perturbed Einstein equation (17); and, in turn, this causes the corresponding evolution of the matter perturbations through Eq. (43). We can write down the appropriate form of the perturbation equation as follows. First, we define the "instantaneous" normalized densities at a cosmic time $t, \tilde{\Omega}_{N}=\rho_{N} / \rho_{c}$, where $\rho_{c}=$ $3 H^{2} / 8 \pi G$ is the critical density at the same instant of time. (Notice that the new densities equal the previously defined ones, i.e. $\tilde{\Omega}_{N}=\Omega_{N}$, only at $t=t_{0}$.) Making use of the definition of the relative DM fluctuation $\delta_{M}$ and of the nonadiabatic perturbed pressure (39), we may cast Eq. (17) in the following way:

$$
\begin{aligned}
& \hat{h}^{\prime}+\frac{2}{a} \hat{h}-\frac{3 H}{a} \tilde{\Omega}_{M} \delta_{M} \\
& =\frac{3 H}{a} \tilde{\Omega}_{D}\left[\left(1+3 c_{s}^{2}\right) \delta_{D}+9 a^{2} H\left(c_{s}^{2}-c_{a}^{2}\right) \frac{\theta_{D}}{k^{2}}\right] .
\end{aligned}
$$

Next we use Eq. (43) to eliminate $\hat{h}$ from (46). With the help of

$$
\begin{gathered}
H^{\prime}(a)=-\frac{4 \pi G}{a H(a)}\left[\rho_{M}(a)+\left(1+w_{e}(a)\right) \rho_{D}(a)\right] \\
=-\frac{3 H(a)}{2 a}\left[1+\frac{w_{e}(a) r(a)}{1+r(a)}\right]
\end{gathered}
$$

and

$$
\tilde{\Omega}_{M}(a)=\frac{1}{1+r(a)}, \quad \tilde{\Omega}_{D}(a)=\frac{r(a)}{1+r(a)},
$$

where $r(a)$ is the cosmic coincidence ratio (1) between the $\mathrm{DE}$ and matter densities, we may finally rewrite (46) as follows:

$$
\begin{aligned}
& \delta_{M}^{\prime \prime}(a)+\frac{3}{2}\left[1-\frac{w_{e}(a) r(a)}{1+r(a)}\right] \frac{\delta_{M}^{\prime}(a)}{a}-\frac{3}{2} \frac{1}{1+r(a)} \frac{\delta_{M}(a)}{a^{2}} \\
& =\frac{3}{2} H(a) \frac{r(a)}{1+r(a)}\left[\left(1+3 c_{s}^{2}\right) \frac{\delta_{D}(a)}{a^{2}}+9\left(c_{s}^{2}-c_{a}^{2}\right) \frac{\theta_{D}(a)}{k^{2}}\right] .
\end{aligned}
$$

If we would neglect the DE fluctuations $\left(\delta_{D}=0, \theta_{D}=0\right)$, the r.h.s. of the previous equation would vanish. Under these conditions, one would be left with a decoupled, 
second-order, homogeneous differential equation that determines the matter perturbations $\delta_{M}$. As could be expected, the resulting equation coincides with Eq. (2.16) of Ref. [23], where the approximation of neglecting the DE perturbations was made right from the start as an intermediate procedure to investigate the amount of linear growth of the matter perturbations and put constraints on the parameter space of the $\Lambda \mathrm{XCDM}$ model. This procedure was called "effective" in that reference, since all the information about the DE is exclusively encoded in the nontrivial EOS function $w_{e}=w_{e}(a)$. Let us write the homogeneous equation as follows:

$$
\delta_{M}^{\prime \prime}(a)+\frac{3}{2}\left(1-w_{e} \tilde{\Omega}_{D}\right) \frac{\delta_{M}^{\prime}(a)}{a}-\frac{3}{2}\left(1-\tilde{\Omega}_{D}\right) \frac{\delta_{M}(a)}{a^{2}}=0,
$$

and let us assume a time interval not very large such that $\tilde{\Omega}_{D}$ and $w_{e}$ remain approximately constant. Looking for a power-law solution of (51) in the limit $\tilde{\Omega}_{D} \ll 1$, we find, for the growing mode,

$$
\delta_{M} \sim a^{1-3\left(1-w_{e}\right) \tilde{\Omega}_{D} / 5} \sim a\left[1-\frac{3\left(1-w_{e}\right)}{5} \tilde{\Omega}_{D} \ln a\right] .
$$

Since $w_{e}<0$ for any conceivable form of $\mathrm{DE}$, this equation shows very clearly that we should expect a growth suppression of matter perturbations (i.e. $\delta_{M} \sim a^{n}$, with $n<1$ ) whenever a (positive) DE density $\tilde{\Omega}_{D}$ is present within the horizon. Physically speaking, we associate this effect to the existence of negative pressure that produces cosmological repulsion of matter.

However, being the DE density nonconstant in general $\left(\delta_{D} \neq 0\right)$, the DE perturbations themselves (and not only the value of the background DE density) should act as a source for the matter fluctuations. This effect is precisely encoded in the inhomogeneous part of Eq. (50), i.e. in its r.h.s which is, in general, nonzero for $\delta_{D}, \theta_{D} \neq 0$. In order to better appreciate this effect, let us consider another simplified situation where the analytical treatment is still possible, namely, let us assume an adiabatic regime $\left(c_{s}^{2}=\right.$ $c_{a}^{2}$ ) with roughly constant $\operatorname{EOS}\left(w_{e} \simeq\right.$ const.) at very large scales (for which $k$ in Eq. (45) is very small, and hence the $\theta_{D}$ component becomes negligible). Under these conditions, Eq. (44) greatly simplifies as follows:

$$
\delta_{D}^{\prime}=\frac{\left(1+w_{e}\right)}{a H} \frac{\hat{h}}{2}=\left(1+w_{e}\right) \delta_{M}^{\prime},
$$

where in the second step we have used (43). The rates of change of the perturbations for matter and DE, therefore, become proportional in this simplified setup. To be more precise, we see from (53) that for $w_{e} \gtrsim-1$ (quintessencelike behavior of the composite DE fluid) the matter fluctuations that are growing with the expansion $\left(\delta_{M}^{\prime}>0\right)$ trigger fluctuations in the DE also growing with the expansion $\left(\delta_{D}^{\prime}>0\right)$, whereas for $w_{e} \lesssim-1$ (phantomlike behavior of the DE) we meet exactly the opposite situation, i.e., increasing fluctuations in the matter density $\left(\delta_{M}^{\prime}>0\right)$ lead to decreasing fluctuations in the $\mathrm{DE}\left(\delta_{D}^{\prime}<0\right)$. Note that upon trivial integration of (53) one finds $\delta_{D}=(1+$ $\left.w_{e}\right) \delta_{M}+C$, where $C$ is a constant determined by the initial conditions. For $C=0$ one obtains a result that fits with the well-known adiabatic initial condition relating the density contrasts of generic matter and DE components [35],

$$
\frac{\delta_{M}}{1+w_{M}}=\frac{\delta_{D}}{1+w_{D}}
$$

where, for nonrelativistic matter, we have $w_{M}=0$, and $\omega_{D}$ is, in this case, the effective EOS $w_{e}$ of the composite DE fluid. Since a positive DE density always leads to cosmological repulsion, it follows from (53) that one should expect some inhibition (resp. enhancement) of the matter growth for the quintessencelike (resp. phantomlike) case.

Although the previous example illustrates the impact of the DE fluctuations on the matter growth for a simple situation, a more complete treatment is required in the general case. In practice, this means that we have to numerically solve the system (43)-(46) or, if desired, replace the last equation with the second-order inhomogeneous equation (50) whose r.h.s. depends on the density contrast and the velocity gradient for the DE, $\delta_{D}, \theta_{D} \neq 0$. Notice that the presence of overdensity DE perturbations $\left(\delta_{D}>0\right)$ does not necessarily imply the inhibition of the corresponding matter perturbations since the coefficient $1+3 c_{s}^{2}$ in front of $\delta_{D}$ on the r.h.s. of Eq. (50) is positive for nonadiabatic DE perturbations. Only for $c_{s}^{2}=c_{a}^{2}$ we meet the aforementioned possibility because $c_{a}^{2} \simeq w_{e}$ is usually negative, unless $w_{e}$ is rapidly decreasing with the expansion, see Eq. (40). In this sense, the discussion above, based on Eq. (53), is only valid at very large scales, specifically for $k$-modes whose length scale $\ell \sim k^{-1}$ is outside the sound horizon (cf. Sec. V). ${ }^{5}$ However, at smaller scales, especially at scales inside the sound horizon, and for a general nonadiabatic regime, we need to solve the aforesaid complete system of equations for the basic set of perturbation variables for the metric, matter, and DE:

$$
\left(\hat{h}(a, k), \delta_{M}(a, k), \delta_{D}(a, k), \theta_{D}(a, k)\right)
$$

In this way, we have extended the effective treatment of the DE perturbations presented in Ref. [23], and we are now ready to better assess the scope of its applicability. In Sec. VII, we will apply this general formalism to the $\Lambda$ XCDM model.

\footnotetext{
${ }^{5}$ In Sec. IX, we will see that this particular situation does accommodate very well the realistic dynamics of the cosmic perturbations for matter and DE during the early stages of the evolution.
} 


\section{SOME GENERIC FEATURES OF THE DE PERTURBATIONS}

In the present section, we summarize some characteristic features of the DE perturbations. Many properties which are, in principle, common to any model with a self-conserved DE, will be later exemplified in Sec. IX within the nontrivial context of the so-called $\Lambda \mathrm{XCDM}$ model [17,23].

\section{A. Divergence at the $\mathrm{CC}$ boundary}

In general, the EOS of the DE will be a dynamical quantity, $w_{e}=w_{e}(a)$. In many models, the EOS may change from quintessencelike $\left(-1<w_{e}<-1 / 3\right)$ to phantom $\left(w_{e}<-1\right)$ behavior or vice versa, acquiring therefore the value $w_{e}=-1$ (also referred to as the "CC boundary") at some instant of time. This is problematic since, as we shall see next, the equations for the perturbations diverge at that point.

The divergence at the $\mathrm{CC}$ boundary is common to any DE model and has been thoroughly studied in the literature (see e.g. $[45,46])$. The problem can be readily seen by direct inspection of Eqs. (44) and (45). Note that, even though $c_{a}^{2}$ diverges at the crossing [cf. (40)], the combination $\left(1+w_{e}\right) c_{a}^{2}$ remains finite and, therefore, Eq. (44) is well behaved. Thus, the problem lies exclusively in the $\left(1+w_{e}\right)$ factor in the denominator of (45). One might think that the divergence can be absorbed through a redefinition of the variables, but this is not the case.

Getting around this difficulty is not always possible. It is well known that there is no way for a single scalar field model to cross the CC boundary [45]. The simplest way to avoid this problem is to assume two fields $(Q, P)$, e.g. one that works as quintessence $w_{Q}>-1$ and dominates the DE density until the CC-crossing point, and beyond it the other field retakes the evolution with a phantom behavior $w_{P}<-1$, or the other way around; see $[46,47]$ for a detailed discussion and specific parametrizations of $w_{e}$. As we will see, in the $\Lambda \mathrm{XCDM}$ model the additional restrictions needed to avoid this divergence will further constrain the physical region of the parameter space.

\section{B. Unbounded growth for adiabatic DE perturbations}

Another well-known problem is the unbounded growth of the DE perturbations for a negative squared speed of sound $c_{s}^{2}$. As already mentioned in the previous section, in the adiabatic case we have $c_{a}^{2} \simeq w_{e}$, which is in general negative as long as the EOS parameter is not varying too fast. As a result, the adiabatic DE perturbations may lead to explosive growth unless extra degrees of freedom are assumed (see e.g. [44] for discussion).

In order to better see the origin of the problem, let us rewrite Eqs. (44) and (45) in terms of conformal time $\eta$, which is easily done by making use of $\dot{f}=a^{2} H f^{\prime}$ (for any $f$ ):

$$
\begin{aligned}
& \dot{\delta}_{D}=-a\left(1+w_{e}\right)\left\{\left[1+\frac{9 \mathcal{H}^{2}\left(c_{s}^{2}-c_{a}^{2}\right)}{k^{2}}\right] \theta_{D}-\frac{\hat{h}}{2}\right\} \\
&-3 \mathcal{H}\left(c_{s}^{2}-w_{e}\right) \delta_{D}, \\
& \dot{\theta}_{D}=-\mathcal{H}\left(2-3 c_{s}^{2}\right) \theta_{D}+\frac{k^{2}}{a} \frac{c_{s}^{2} \delta_{D}}{\left(1+w_{e}\right)} .
\end{aligned}
$$

As in Sec. III, we have defined $\mathcal{H}=\dot{a} / a \equiv H a$. If we use the two equations above and Eq. (46) to get a second-order differential equation for $\delta_{D}$, we arrive at

$$
\ddot{\delta}_{D}=-k^{2} c_{s}^{2} \delta_{D}+\mathcal{O}\left(\hat{h}, \delta_{M}, \theta_{D}, \delta_{D}\right),
$$

where the second term on the r.h.s. represents other terms linear in these variables. Assuming that the various perturbations are initially more or less of the same order, we see that the first term on the r.h.s. of (58) will dominate provided

$$
\left|k \int c_{s} d \eta\right| \gg 1
$$

Notice that, for constant sound velocity, this condition simply tells us that the wavelength of the modes satisfies $\ell \sim k^{-1} \ll \lambda_{s}$, where $\lambda_{s}=c_{s} \eta$ is the "sound horizon." Equation (59) is a generalization of this condition for arbitrary sound speed, in which case the sound horizon is given by

$$
\lambda_{s}=\int_{0}^{\eta} c_{s} d \eta=\int_{0}^{a} \frac{c_{s} d \tilde{a}}{\tilde{a}^{2} H(\tilde{a})}
$$

and constitutes a characteristic scale for the DE perturbations. As we will see next, the DE is expected to be smooth for scales well below it $[43,48]$.

For scales well inside the sound horizon, (58) becomes the equation of a simple harmonic oscillator, whose solution is (in what follows, we assume constant $c_{s}^{2}$ for simplicity):

$$
\delta_{D}=C_{1} e^{i c_{s} k \eta}+C_{2} e^{-i c_{s} k \eta},
$$

where $C_{1}$ and $C_{2}$ are constants. We see that, for $c_{s}^{2}<0$ (i.e. imaginary $c_{s}$ ) and neglecting the decaying mode, the DE perturbations grow exponentially. Obviously, this situation is unacceptable for structure formation. ${ }^{6}$ On the other hand, if $c_{s}^{2}>0$ the DE density contrast oscillates. Since $\delta_{M}$ grows typically as the scale factor $a$, this ensures that the ratio $\delta_{D} / \delta_{M} \sim \delta_{D} / a \rightarrow 0$ with the expansion. In other

\footnotetext{
${ }^{6}$ Let us note that this kind of problem need not occur when the $\mathrm{DE}$ is a pure running $\Lambda$, for then the perturbation $\delta \rho_{\Lambda}$ is no longer an independent dynamical variable and is, in fact, determined by an algebraic function of the other perturbation variables, see [41] for details.
} 
words, this tells us that the DE is going to be a smooth component (as it is usually assumed) as long as we are well inside the sound horizon. This feature is treated in more depth in the following section.

\section{Smoothness of DE below the sound horizon}

As a matter of fact, Eq. (58) is an oversimplification. In addition to having a term proportional to $\delta_{D}$, we also have one depending on its first derivative. So we can write that equation more precisely as follows:

$$
\ddot{\delta}_{D}=D_{1} \delta_{D}+D_{2} \dot{\delta}_{D}+\mathcal{O}\left(\hat{h}, \delta_{M}, \theta_{D}\right),
$$

which gives us not just a simple, but a damped harmonic oscillator. The coefficients $D_{1}$ and $D_{2}$ are, in general, functions of the conformal time $\eta$. So the DE density contrast does not only oscillate, but its amplitude decreases with time. Indeed, it was shown in [43] that the quantity

$$
\delta_{D}^{\text {(rest) }}=\delta_{D}+3 \mathcal{H} a\left(1+w_{e}\right) \frac{\theta_{D}}{k^{2}},
$$

which corresponds to the density contrast in the DE rest frame, oscillates with an amplitude $A$ that decreases according to

$$
A \propto c_{s}^{-1 / 2} a^{\left(-1+3 w_{e}\right) / 2} .
$$

The damped oscillations of the DE density contrast are clearly seen in the $\Lambda \mathrm{XCDM}$ model, as we will show in Sec. IX.

Finally, we may ask ourselves whether the scales relevant to the LSS surveys [4] are well inside the sound horizon or not. Note that, in a matter-dominated universe with negligible CC term and constant $c_{s}$, we have $H^{2}=$ $H_{0}^{2} \Omega_{M}^{0} a^{-3}$ and the sound horizon (60) takes the simple form

$$
\lambda_{s}=\frac{2 c_{s}}{H_{0}\left(\Omega_{M}^{0}\right)^{1 / 2}} \sqrt{a}
$$

Thus, in general, we expect that the size of the sound horizon at present $\left(a_{0}=1\right)$ should be roughly of the order of the Hubble length $H_{0}^{-1}$. On the other hand, the observational data concerning the linear regime of the matter power spectrum lie in the range $0.01 h \mathrm{Mpc}^{-1}<k<$ $0.2 h \mathrm{Mpc}^{-1}$ [4], which corresponds to length scales $\ell \sim$ $k^{-1}$ comprised in the interval $\left(600 H_{0}\right)^{-1}<\ell<\left(30 H_{0}\right)^{-1}$, hence well below the sound horizon (at least for $c_{s}^{2}$ not too close to 0). Therefore, according to the previous discussions, we expect the DE density to be smooth at those scales, and indeed it will be so for the $\Lambda$ XCDM model. Nevertheless, as we will see in Sec. IX, the larger the scale $\ell$ or the smaller the speed of sound $c_{s}$, the more important the DE perturbations are, because then (59) is not such a good approximation.

\section{THE $\Lambda$ XCDM MODEL AS A CANDIDATE TO} SOLVE THE COSMIC COINCIDENCE PROBLEM

The $\Lambda$ XCDM model [17] provides an interesting way of explaining the so-called cosmological coincidence problem (cf. Sec. II). The idea is related to the possibility of having a dynamical component $X$, called the "cosmon," which interacts with a running cosmological constant $\Lambda$. If the matter components are canonically conserved, the composite DE "fluid" made out of $X$ and the running $\Lambda$ will be a self-conserved medium too. The dynamics of the $\Lambda \mathrm{XCDM}$ universe is such that its composite DE may enforce the existence of a stopping point after many Hubble times of cosmological expansion. As a result, this modified FLRW-like universe can remain for a long while in a situation where the coincidence ratio (1) does not change substantially from the time when the DE became significant until the remote time in the future when the stopping point is attained. Subsequently, the Universe reverses its motion till the big crunch.

The total DE density and pressure for the $\Lambda \mathrm{XCDM}$ universe are obtained from the sum of the respective $C C$ and $X$ components:

$$
\rho_{D}=\rho_{\Lambda}+\rho_{X}, \quad p_{D}=p_{\Lambda}+p_{X} .
$$

The evolving CC density $\rho_{\Lambda}(t)=\Lambda(t) / 8 \pi G$ of the model is motivated by the quantum field theory formulation in curved space-time by which the $\mathrm{CC}$ is a solution of a renormalization group equation. Following $[13,15,16,19,49]$, the $\mathrm{CC}$ density emerges in general as a quadratic function of the expansion rate:

$$
\rho_{\Lambda}(H)=\rho_{\Lambda}^{0}+\frac{3 \nu}{8 \pi} M_{P}^{2}\left(H^{2}-H_{0}^{2}\right)
$$

where $\rho_{\Lambda}^{0}=\rho_{\Lambda}\left(H=H_{0}\right)$ is the present value. The dimensionless parameter $\nu$ is given by

$$
\nu \equiv \frac{\sigma}{12 \pi} \frac{M^{2}}{M_{P}^{2}},
$$

where $M$ is an effective mass parameter representing the average mass of the heavy particles of the grand unified theory (GUT) near the Planck scale, after taking into account their multiplicities. Depending on whether they are bosons or fermions, $\sigma=+1$ or $\sigma=-1$, respectively. For example, for $M=M_{P}$ one has $|\nu|=\nu_{0}$, where

$$
\nu_{0} \equiv \frac{1}{12 \pi} \simeq 2.6 \times 10^{-2} .
$$

On physical grounds, we expect that this value of $|\nu|$ should be the upper bound for this parameter. In the next

\footnotetext{
${ }^{7}$ Originally, the cosmon appeared in Ref. [7] as a scalar field linked to the mechanism of dynamical adjustment of the CC. In the present context, the entity $X$ is also differentiated from the $\mathrm{CC}$, but if taken together they form a composite and interactive DE medium.
} 
section, we will see if we can pinpoint a region of parameter space compatible with this expectation.

The energy density associated to the cosmon component $X$ is obtained from the total DE conservation law (36) and the composite form (66),

$$
\rho_{X}^{\prime}(a)+\rho_{\Lambda}^{\prime}(a)=-\frac{3}{a}\left(1+w_{X}\right) \rho_{X}(a),
$$

where $w_{X}$ is the effective EOS parameter of $X$,

$$
p_{X} \equiv w_{X} \rho_{X}
$$

In principle, $w_{X}$ could be a function of the scale factor. However, a simpler assumption that allows us to perform a completely analytic treatment is to consider that the $X$ component behaves as a barotropic fluid with a constant EOS parameter in one of the following two ranges: $\omega_{X} \gtrsim$ -1 (quintessencelike cosmon) or $\omega_{X} \lesssim-1$ (phantomlike cosmon). On the other hand, the EOS parameter for the running $\Lambda$ component still remains as the cosmological constant one, $w_{\Lambda}=-1$, i.e.

$$
p_{\Lambda} \equiv-\rho_{\Lambda} \text {. }
$$

From these assumptions, it is easy to find the following relation between the effective EOS parameter of the total DE fluid (37) and the EOS parameter of the cosmon $w_{X}$ :

$$
\left(1+w_{e}(a)\right) \rho_{D}(a)=\left(1+w_{X}\right) \rho_{X}(a) .
$$

The normalized density of the cosmon component, $\Omega_{X}(a)=\rho_{X}(a) / \rho_{c}^{0}$, can be obtained from the previous relations after solving the differential equation (70). In this equation, we have $\rho_{\Lambda}^{\prime}(a)=(3 \nu / 8 \pi) M_{P}^{2} d H^{2} / d a$ from (67), and the derivative $d H^{2} / d a=2 H(a) H^{\prime}(a)$ can be explicitly computed from (47) upon using (35) and (73). One finally obtains the differential equation

$$
\Omega_{X}^{\prime}(a)+\frac{3}{a}\left(1+\omega_{X}-\epsilon\right) \Omega_{X}(a)=3 \nu \Omega_{M}^{0} a^{-4} .
$$

With the boundary condition that the current value of $\rho_{X}$ is $\rho_{X}^{0}$, the solution of (74) can be written in the following way $^{8}$ :

$$
\Omega_{X}(a)=\Omega_{X}^{0}\left[(1+b) a^{-3\left(1+w_{X}-\epsilon\right)}-b a^{-3}\right],
$$

where in the above equations we have used the notations

$$
\begin{gathered}
b \equiv-\frac{\nu \Omega_{M}^{0}}{\left(\omega_{X}-\epsilon\right) \Omega_{X}^{0}}, \\
\epsilon \equiv \nu\left(1+w_{X}\right) .
\end{gathered}
$$

\footnotetext{
${ }^{8}$ The fact that the evolution of the cosmon $X$ is completely determined by the dynamics of the running $\rho_{\Lambda}$ (67), together with the hypothesis of total DE conservation (70), implies that $X$ cannot be generally assimilated to a scalar field, which has its own dynamics. In fact, as we have already mentioned, $X$ is to be viewed in general as an effective entity within the context of the effective action of QFT in curved space-time.
}

As we will discuss in more detail below, the parameter $\epsilon$ must remain small $(|\epsilon|<0.1)$ in order to be compatible with primordial nucleosynthesis. For $\nu=0$ the $\mathrm{CC}$ density (67) becomes constant. In this case, the two parameters (76) and (77) vanish and Eq. (75) boils down to the simplest possible form, which is characteristic of a selfconserved monocomponent system,

$$
\Omega_{X}(a)=\Omega_{X}^{0} a^{-3\left(1+w_{X}\right)} .
$$

It is only in this particular situation where the cosmon $X$ could be a self-conserved scalar field with its own dynamics. But in general this is not so because in QFT in curved space-time we have good reasons to expect a running $\rho_{\Lambda}$ $[13,15,16]$, and hence $\nu \neq 0$. Therefore, if the total DE is to be conserved, the dynamics of $X$ is not free anymore and becomes determined as in (75).

The normalized total DE density $\Omega_{D}=\rho_{D} / \rho_{c}^{0}$ is given by

$$
\begin{aligned}
\Omega_{D}(a)= & \left(\frac{1-\Omega_{\Lambda}^{0}}{1-\nu}-\frac{w_{X} \Omega_{M}^{0}}{w_{X}-\epsilon}\right) a^{-3\left(1+w_{X}-\epsilon\right)}+\frac{\Omega_{\Lambda}^{0}-\nu}{1-\nu} \\
& +\frac{\epsilon \Omega_{M}^{0} a^{-3}}{w_{X}-\epsilon}
\end{aligned}
$$

where the various current normalized densities satisfy the relation $\Omega_{M}^{0}+\Omega_{D}^{0}=\Omega_{M}^{0}+\Omega_{\Lambda}^{0}+\Omega_{X}^{0}=1$, which may be called the " $\Lambda \mathrm{XCDM}$ cosmic sum rule." Using (73), the effective EOS of the DE in the $\Lambda$ XCDM model can now be obtained explicitly,

$$
w_{e}(a)=-1+\left(1+\omega_{X}\right) \frac{\Omega_{X}(a)}{\Omega_{D}(a)},
$$

with $\Omega_{X}(a)$ and $\Omega_{D}(a)$ given by (75) and (79), respectively.

The total DE density (79) varies in such a way that the ratio (1) can remain under control, which is the clue for solving the coincidence problem in a dynamical way [17]. Indeed, the explicit computation of such a ratio yields

$$
\begin{aligned}
r(a)= & {\left[\frac{1-\Omega_{\Lambda}^{0}}{\Omega_{M}^{0}(1-\nu)}-\frac{w_{X}}{w_{X}-\epsilon}\right] a^{-3\left(w_{X}-\epsilon\right)}+\frac{\left(\Omega_{\Lambda}^{0}-\nu\right) a^{3}}{(1-\nu) \Omega_{M}^{0}} } \\
& +\frac{\epsilon}{w_{X}-\epsilon},
\end{aligned}
$$

and it can be bounded due to the existence of a maximum (triggered by the $\sim a^{-3\left(w_{X}-\epsilon\right)}$ term in the previous formula). Moreover, $r(a)$ given above stays relatively constant (typically not varying more than 1 order of magnitude) for a large fraction of the history of the Universe and for a significant region of the parameter space [17]. In contrast, in the standard concordance $\Lambda \mathrm{CDM}$ model, the CC density remains constant, $\rho_{\Lambda}=\rho_{\Lambda}^{0}$, and the coincidence ratio grows unstoppably with the cubic power of the scale factor, $r(a)=\Omega_{\Lambda}^{0} a^{3} / \Omega_{M}^{0}$. In this scenario, it is difficult to explain why the constant $\rho_{\Lambda}=\rho_{\Lambda}^{0}$ is of the same order of magni- 
tude as the matter density right now: $\rho_{M}^{0}$. Let us point out that the standard model ratio is just that particular case of (81) for which $\nu=0$ (no running CC) and $\Omega_{X}^{0}=0$ (no cosmon).

Before closing this section, we would like to make a remark and some discussion concerning the quadratic evolution law (67) for the cosmological term. This equation was originally motivated within the framework of the renormalization group (RG) of QFT in curved space-time $[12-14,18,49]$ (see also [15] for a short review). We point out a criticism against this approach that recently appeared in the literature [50]. While the nature of this criticism was amply rejected in [16] (see below for a summary), it is fair to say that the question of whether a rigorous RG approach in cosmology is feasible is still an open question and remains a part of the $\mathrm{CC}$ problem itself. Although it is not our main aim here to focus on this fundamental issue, let us briefly sketch the situation along the lines of Ref. [16], to which we also refer the reader for a summary of the rich literature proposing different RG formulations of cosmology both in QFT in curved space-time and in quantum gravity.

The RG method in cosmology treats the vacuum energy density as a running parameter and aims at finding a fundamental differential relation (renormalization group equation) of the form

$$
\frac{d \rho_{\Lambda}}{d \ln \mu}=\beta_{\Lambda}(P, \mu)
$$

which is supposed to describe the leading quantum contributions to it, where $\beta_{\Lambda}$ is a function of the parameters $P$ of the effective action (EA) and $\mu$ is a dimensional scale. The appearance of this arbitrary mass scale is characteristic of the renormalization procedure in QFT owing to the intrinsic breaking of scale invariance by quantum effects. The quantity $\rho_{\Lambda}$ in (82) is a ( $\mu$-dependent) renormalized part of the complete QFT structure of the vacuum energy. Depending on the renormalization scheme, the scale $\mu$ can have a more or less transparent physical meaning, but the physics should be completely independent of it. Such (overall) $\mu$ independence of the observable quantities is actually the main message of the RG; but, remarkably enough, the $\mu$ dependence of the individual parts is also the clue of the RG technique to uncover the leading quantum effects.

Essential for the RG method in cosmology is to understand that, in order for the vacuum energy to acquire dynamical properties, we need a nontrivial external metric background. The dynamical properties of this curved background (e.g. the expanding FLRW space-time, characterized by the expansion rate $H$ ) are expected to induce a functional dependence $\rho_{\Lambda}=\rho_{\Lambda}(H)$. The latter should follow from parametrizing the quantum effects with the help of the scale $\mu$ and then using some appropriate correspondence of $\mu$ with a physical quantity, typically with $H$ in the cosmological context, although there are other possibilities [12-14]. In this way, one expects to estimate the subset of quantum effects reflecting the dynamical properties of the nontrivial background. Although $\mu$ cancels in the full EA, the RG method enables one to separate the relevant class of quantum effects responsible for the running. The procedure is similar to the $R G$ in a scattering process in $\mathrm{QCD}$; the parametrization of the quantum effects in terms of $\mu$ is the crucial strategy to finally link them with the energy of the process through the correspondence $\mu \rightarrow q$ (where $q$ is a typical momentum of the scattering process) at high energy. One can also proceed in the same way in QED and electroweak theory (although here one can adopt more physical subtraction schemes, if desired). The RG technique can actually be extended to the whole particle physics domain. In cosmology, however, the situation is more complicated, partly because (as remarked above) the physical scale behind the quantum effects is not obvious. Still, one expects that it should be related with the expanding metric background and hence the expansion rate $H$ can be regarded as a reasonable possibility. On this basis, the heuristic arguments exhibited in $[13,14,16,18,19,49]$ combined with the general covariance of the EA suggest that the solution of the RG equation (82) should lead to the kind of quadratic law (67) that we have used.

According to [16], the point of view of Ref. [50] is incorrect on two main accounts: first, because they try to disprove the running through the overall cancellation of the arbitrary scale $\mu$ in the EA; and second, because they neglect the essential role played by the nontrivial metric background. As emphasized in [16], the cancellation of $\mu$ in the EA cannot be argued as a valid criticism because this fact is a built-in feature of the RG and it was never questioned. If this would be a real criticism, it would also apply to QED, QCD, or any other renormalizable QFT, and nevertheless this is no obstacle for using the RG method in these theories as an extremely useful strategy to extract the dependence of the quantum effects on the physical energy scale of the processes, in particular, the socalled running coupling constants $g_{s}=g_{s}(q)$ and $e=e(q)$ of the strong and electromagnetic interactions. Moreover, in the absence of a nontrivial metric background, there is no physical running of the vacuum energy, even though there is still $\mu$ dependence of the various parts of the EA and, in particular, of the CC, see e.g. [51]. Therefore, at the end of the day such criticism seems to go against the essence of the RG method and its recognized ability to encapsulate the leading quantum effects on the physical observables.

In cosmology, the principles of the RG should be the same, but there are two main stumbling blocks that prevent from straightforwardly extending the method in practice [16], to wit: (i) the aforesaid lack of an obvious/unique correspondence of $\mu$ with a cosmological scale defining 
the physical running, and also (no less important) (ii) the huge technical problems related with the application of the RG within a physical (momentum-dependent) renormalization scheme in a curved background. These difficulties are unavoidable here because we are dealing with QFT in the infrared regime and moreover the metric expansions cannot be performed on a flat background; indeed, there cannot be a flat background in the presence of a cosmological term. While these two problems remain unresolved in a completely satisfactory manner, it is legitimate to use the phenomenological approach and the educated guess (e.g. the general covariance of the EA) to hint at the running law. This is the guiding principle followed in the aforementioned references and that led to Eq. (67).

Finally, let us emphasize that irrespective of whether such a law can be substantiated within the strict framework of the RG, the present study remains perfectly useful simply treating (67) as an acceptable type of a phenomenological variation law and keeping also in mind that adding the cosmon may contribute to the resolution of the pressing cosmic coincidence problem.

\section{DARK ENERGY PERTURBATIONS IN THE $\triangle$ XCDM MODEL}

In this section, we further elaborate on the conditions to bound the ratio $r=r(a)$ and discuss the constraints on the parameter space, in particular, the impact of the DE perturbations on these constraints. In Sec. V, we discussed analytically some generic features about $\mathrm{DE}$ perturbations. In principle, those results should apply to any model in which the DE is self-conserved. The $\Lambda \mathrm{XCDM}$ model, given its peculiarities (a composite DE which results in a complicated evolution of the effective EOS), constitutes a nontrivial example of that kind of model. In this sense, it is interesting to use the $\Lambda \mathrm{XCDM}$ to put our general predictions to the test. At the same time, this will allow us to impose new constraints on the parameter space of the model, improving its predictivity.

The parameter space of the $\Lambda$ XCDM model was already tightly constrained in [23]. In that work, the matter density fluctuations were analyzed under the assumption that the DE perturbations could be neglected. As a first approximation this is reasonable since, as we have discussed in Sec. V, the DE is expected to be smooth at scales well below the sound horizon. Thus, we will take the results of [23] as our starting point and will check numerically the goodness of that approximation. Finally, we will further constrain the parameter space using the full approach presented in the present work. Let us summarize the constraints that were imposed in [23]:

(1) Nucleosynthesis bounds: As already commented, the ratio (81) between $\mathrm{DE}$ and matter densities should remain relatively small at the nucleosynthesis time, in order not to spoil the big bang model predictions on light-element abundances. Requiring that ratio to be less than $10 \%$, it roughly translates into the condition $|\epsilon|<0$.1, where the parameter $\epsilon$ was defined in Eq. (77).

(2) Solution of the coincidence problem: In Ref. [17], where the $\Lambda \mathrm{XCDM}$ model was originally introduced as a possible solution to the coincidence problem, it was shown that there is a large subvolume of the total $\triangle \mathrm{XCDM}$ parameter space for which the ratio $r(a)$ remains bounded and near the current value $r_{0}$ (say, $|r(a)| \lesssim 10 r_{0}$, where $r_{0} \sim$ $7 / 3$ ) during a large fraction of the history of the Universe. Thus, the fact that the matter and DE densities are comparable right now may no longer be seen as a coincidence. Such a solution of the coincidence problem is related to the existence of a future stopping (and subsequent reversal) of the Universe expansion within the relevant region of the parameter space.

(3) Current value of the EOS parameter: Recent studies (see e.g. [2]) suggest that the value of the DE effective EOS should not be very far from -1 at present. Although these results usually rely on the assumption of a constant EOS parameter (and thus are not directly applicable to the $\Lambda$ XCDM model), we adopted a conservative point of view and stuck to them by enforcing the condition $\left|1+w_{e}(a=1)\right| \leq$ 0.3 on the EOS function (80).

(4) Consistency with LSS data: As said before, in [23] we studied the growth of matter density fluctuations under the assumption that the DE was smooth on the scales relevant to the linear part of the matter power spectrum. From the fact that the standard $\Lambda \mathrm{CDM}$ model provides a good fit to the observational data, we took it as a reference and imposed that the amount of growth (specifically, the matter power spectrum), of our model did not deviate by more than $10 \%$ from the $\Lambda \mathrm{CDM}$ value ("F-test" condition). This condition can also be justified from the observed galaxy fluctuation power spectrum, see [23] for more details.

The upshot of that analysis was that there is still a big subvolume of the three-dimensional $\Lambda \mathrm{XCDM}$ parameter space $\left(\nu, \omega_{X}, \Omega_{\Lambda}^{0}\right)$ satisfying simultaneously the above conditions. ${ }^{9}$ The projections of that volume onto the three perpendicular planes $\left(\nu, \Omega_{\Lambda}^{0}\right),\left(\nu, \omega_{X}\right)$, and $\left(\Omega_{\Lambda}^{0}, \omega_{X}\right)$ are displayed in Figs. 1 and 2 (shaded regions). These regions where already determined in Ref. [23]. In the next section, we will discuss how the final set of allowed points becomes further reduced when we take into account the analysis of the DE perturbations.

\footnotetext{
${ }^{9}$ In Ref. [23], we took a prior for the normalized matter density at present, specifically $\Omega_{M}^{0}=0.3$. This means that $\Omega_{D}^{0}=0.7$ for a spatially flat universe. For better comparison with those results, we keep this prior also in the present work.
} 


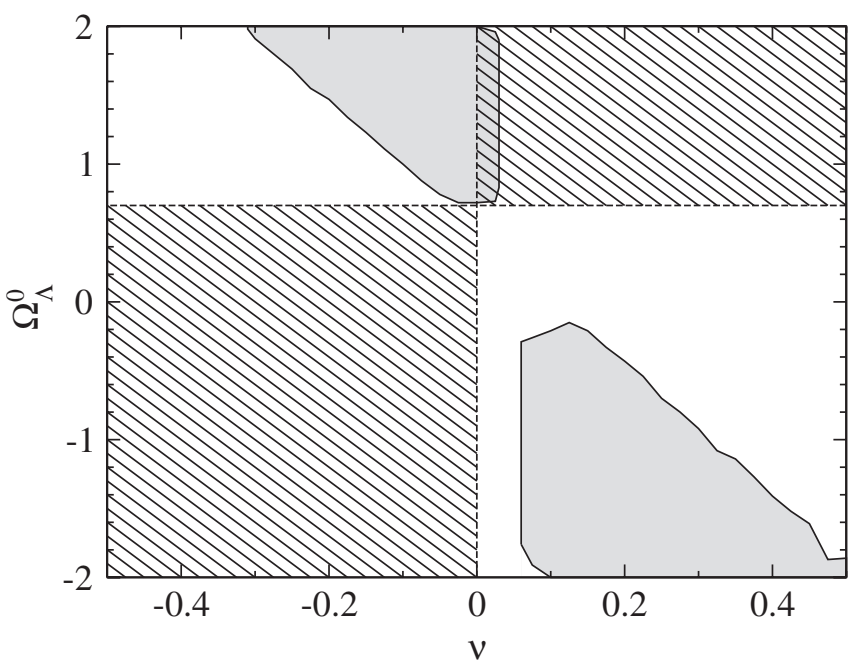

FIG. 1. Projection of the 3D physical volume of the $\Lambda \mathrm{XCDM}$ model onto the $\nu-\Omega_{\Lambda}^{0}$ plane. Shaded area: points that satisfy all the constraints in [23], see also Sec. VII of the present work. Striped area: points that are not affected by the divergence at the $\mathrm{CC}$ boundary discussed in Sec. VII A. The final allowed region is the one both shaded and striped. As a result of considering the DE perturbations, the possible values of the parameters become strongly restricted, which implies a substantial improvement in the predictive power of the model.

\section{A. Divergent behavior at the $\mathrm{CC}$ boundary}

As discussed in Sec. V, if the effective EOS of the model crosses the CC boundary $\left(w_{e}=-1\right)$ at some point in the past, the perturbation equations will present a real divergence. Obviously, this circumstance makes the numerical analysis unfeasible at the points of parameter space affected by the singularity.

In the absence of an apparent mechanism to get around this singularity, we are forced to restrict our parameter space to the subregion where the solution of the perturbation equations (43)-(46) is regular, namely, by removing those points of the parameter space that present such a crossing in the past, because these points cannot belong to a well-defined history of the Universe. In the absence of a more detailed definition of the cosmon entity $X$, this new constraint is unavoidable. This should not be considered as a drawback of the model, for even in the case when one uses a collection of elementary scalar fields to represent the $\mathrm{DE}$, one generally meets the same kind of divergent behavior as soon as the $\mathrm{CC}$ boundary is crossed, unless some special conditions are arranged. In other words, even if the components of the DE are as simple as, say, elementary scalar fields with smooth behavior and well-defined dynamical properties (including an appropriately chosen potential), there is no a priori guarantee that the $\mathrm{CC}$ boundary can be crossed safely [45]. It is possible to concoct ingenious recipes, see e.g. [46], such that the perturbation equations become regular at the $\mathrm{CC}$ boundary, but the procedure is artificial in that one must introduce new fields (one
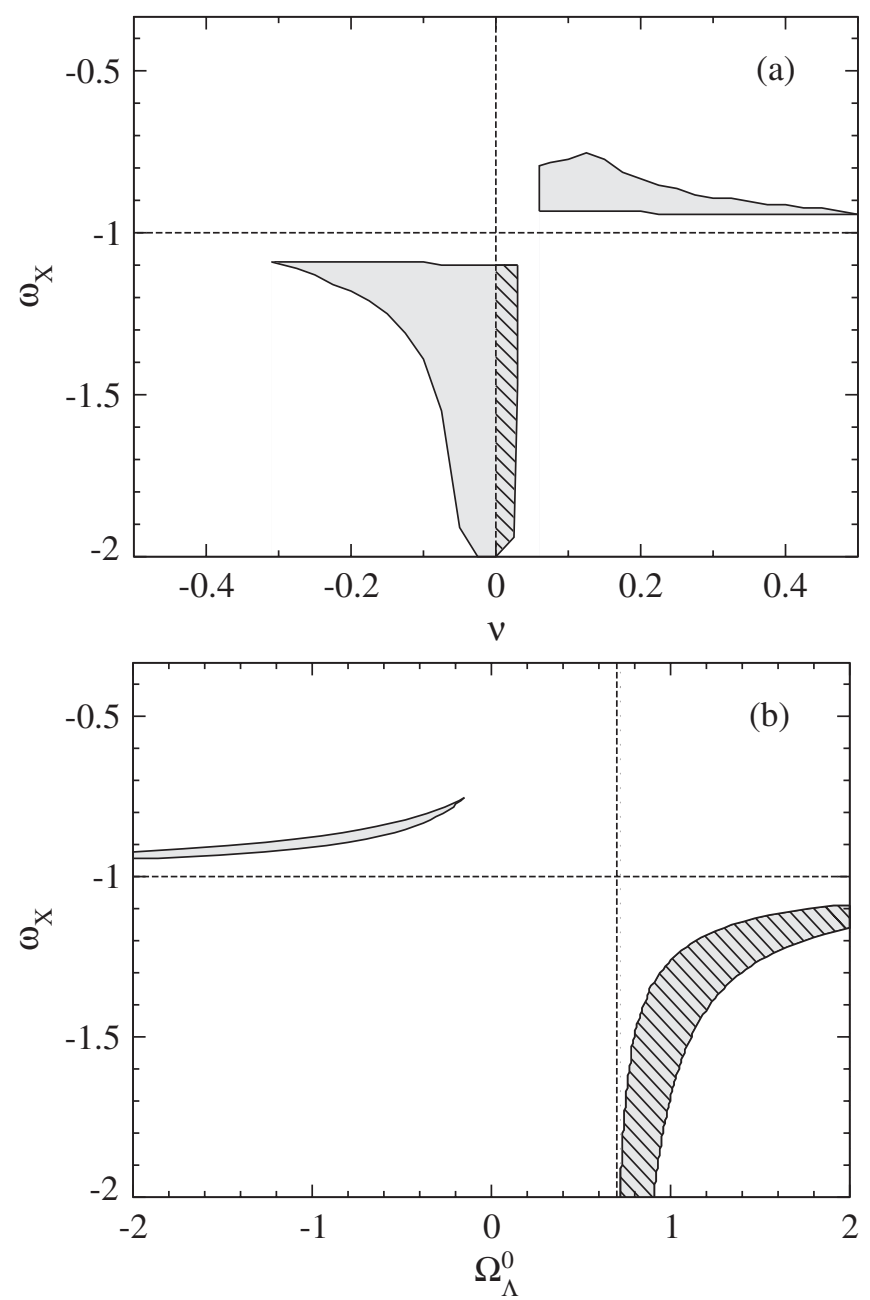

FIG. 2. Projection of the 3D physical volume of the $\Lambda \mathrm{XCDM}$ model onto the $\nu-\omega_{X}$ and $\Omega_{\Lambda}^{0}-\omega_{X}$ planes. Shaded: points that satisfy all the constraints in [23]. Striped: points that, in addition, are not affected by the divergence at the $\mathrm{CC}$ boundary discussed in Sec. VII A. The final allowed region is the one both shaded and striped.

quintessencelike and another phantomlike) satisfying special properties such that their respective EOS behaviors match up continuously at the CC crossing. Apart from the fact that fields with negative kinetic terms are not very welcome in QFT, one cannot just replace the original fields with the new ones without at the same time changing the original DE model. As we will see below, in the $\Lambda$ XCDM case the absence of $\mathrm{CC}$ crossing projects out a region of the parameter space which is significantly more reduced, and therefore the predictive power of the model becomes substantially enhanced.

In Sec. 6 of the first reference in [17], it was shown that the necessary and sufficient condition for having a CCboundary crossing in the past within the $\Lambda$ XCDM model is that the parameter $b$ given in (76) is positive. As can be readily seen, this will happen whenever $\nu$ and $\Omega_{X}^{0}$ have the same sign (where we use the fact that $\omega_{X}<0$ and 
$|\epsilon| \ll\left|\omega_{X}\right|$ in the relevant region of parameter space). From the cosmic sum rule of the $\Lambda$ XCDM model, we have $\Omega_{X}^{0}=1-\Omega_{M}^{0}-\Omega_{\Lambda}^{0}$; thus, using our prior $\Omega_{M}^{0}=$ 0.3 , the set of allowed points (for which $\Omega_{X}^{0}$ has different sign from $\nu$ ) are those comprised in the striped areas in Figs. 1 and 2. This leaves us with a very small allowed region in each plane, which is just the corresponding intersection of the shaded area and the striped one.

At the end of the day, it turns out that most of the points in the shaded area in Figs. 1 and 2 (viz. those allowed by the conditions stated in the previous section and the analysis of [23]) are ruled out by the new constraint emerging from the DE perturbations analysis, and hence we end up with a rather definite prediction for the values of the $\Lambda \mathrm{XCDM}$ parameters. In particular, we find from these figures that only small positive values of $\nu$ are allowed, at most of order $\nu \sim 10^{-2}$. Let us emphasize that this is in very good agreement with the theoretical expectations mentioned in Sec. VI. Recall that, from the point of view of the physical interpretation of $\nu$ in Eq. (68), we expected $\nu$ in the ballpark of $\nu_{0} \sim 10^{-2}$ at most-see Eq. (69) since the masses of the particles contributing to the running of the CC should naturally lie below the Planck scale. ${ }^{10}$ Let us mention that the interesting bounds on $\nu$ obtained in Ref. [52] on the basis of the so-called generalized second law of gravitational thermodynamics would suggest that only the effective mass near the Planck mass is allowed. However, let us point out that such a study has been performed without including the nontrivial effect from the cosmon.

A very important consequence of the dark energy perturbative constraint is that the effective EOS of the DE can be quintessencelike only, i.e. $-1<w_{e}<-1 / 3$. To prove this statement, let us start from Eq. (73). For the current values of the parameters, this equation can be rewritten as

$$
\left(1+w_{e}^{0}\right) \Omega_{D}^{0}=\left(1+\omega_{X}\right) \Omega_{X}^{0},
$$

where $w_{e}^{0} \equiv w_{e}(a=1)$ is the value of the effective EOS parameter at the present time. Looking at Figs. 2(a) and 2(b), we realize the following two relevant features: first, the cosmon component is necessarily phantomlike $\left(\omega_{X}<-1\right)$ in the allowed region by the DE perturbations; and, second, its energy density at present is negative; namely, $\Omega_{X}^{0}=0.7-\Omega_{\Lambda}^{0}<0$ because from Fig. 2(b) we have $\Omega_{\Lambda}^{0}>0.7$. Therefore, since the r.h.s. of (83) is constrained to be positive and $\Omega_{D}^{0}=0.7>0$, we are enforced to have $w_{e}^{0}>-1$. However, the fulfillment of this condition at present implies its accomplishment in the past, i.e.

\footnotetext{
${ }^{10}$ Let us clarify that the tighter bounds on $\nu$ determined in Ref. [41] are possible only because, in the latter work, the DE is not conserved and there is no cosmon. As we have shown in [23], a running cosmological constant model without a self-conserved DE cannot solve the coincidence problem in a natural way because the required values of $\nu$ are too large and, hence, incompatible with the physical interpretation of this parameter.
}

$w_{e}(a)>-1(\forall a \leqslant 1)$, otherwise there would have been a crossing of the CC boundary at some earlier time, which is excluded by the analysis of the DE perturbations. The upshot is that the EOS of the DE in the $\Lambda \mathrm{XCDM}$ model can only appear effectively as quintessence (q.e.d.). In reality, it only mimics quintessence, of course, as its ultimate nature is not; such DE medium is a mixture made out of running vacuum energy and a compensating entity that insures full energy conservation of the compound system.

Dark energy components $X$ with negative energy density are peculiar in cosmology since, in contrast to standard DE components, they satisfy the strong energy condition (like ordinary matter), and as a result the gravitational behavior of $X$ is attractive rather than repulsive. Because of this double resemblance with matter and phantom $\mathrm{DE}$ (although with the distinctive feature $\rho_{X}<0$ ), such components can be called "phantom matter" [17]. Being $X$ in general an effective entity, such "phantom matter" behavior is actually nonfundamental.

\section{B. Adiabatic speed of sound in the $\Lambda \mathrm{XCDM}$}

In the Eqs. (43)-(46), we assumed the most general case in which the perturbations could be nonadiabatic. Moreover, we have shown that the adiabatic case usually leads to an unphysical exponential growth of the perturbations as a result of $c_{a}^{2}$ in (40) being negative. Next we will check that, indeed, the most common situation in the $\Lambda \mathrm{XCDM}$ model is to have $c_{a}^{2}<0$. Notwithstanding, adiabatic perturbations are not completely forbidden in the present framework, as there is a small region of the parameter space for which $c_{a}^{2}$ could be positive.

From Eqs. (40) and (73), and making use of the DE conservation law (36), we find that the adiabatic speed of sound for the $\Lambda \mathrm{XCDM}$ model can be cast as follows:

$$
c_{a}^{2}(a)=-1-\frac{a}{3} \frac{\Omega_{X}^{\prime}(a)}{\Omega_{X}(a)} .
$$

With the help of (75), we can rewrite the last expression as

$$
c_{a}^{2}(a)=\frac{\Omega_{X}^{0}}{\Omega_{X}(a)}(1+b)\left(\omega_{X}-\epsilon\right) a^{-3\left(1+\omega_{X}-\epsilon\right)} .
$$

We want to find out the condition for this expression to be positive. As $\left(\omega_{X}-\epsilon\right)<0$ (remember that $\omega_{X}<0$ and $|\epsilon|<0.1$, due to nucleosynthesis constraints), that condition simply reads

$$
\frac{\Omega_{X}^{0}}{\Omega_{X}(a)}(1+b)<0 .
$$

The cosmon energy density $\Omega_{X}(a)$ cannot vanish because, in such a case, the perturbation equations would diverge. Indeed, $\Omega_{X}(a)=0$ corresponds to a CC-boundary crossing at some value of the scale factor in the past, cf. Eq. (80). Thus, being $\Omega_{X}(a)$ a continuous function, it must have the same sign in the past as that of its present value, i.e. 
$\left.\Omega_{X}^{0} / \Omega_{X}(a)>0(\forall a \leqslant 1)\right)$. In short, the final condition that ensures that $c_{a}^{2}>0$ is

$$
(1+b)<0 .
$$

If this condition would be satisfied, then $c_{a}^{2}>0$ would hold for the entire past history of the Universe and, under these circumstances, the adiabatic equations may be used. It turns out that the relation (87) can be satisfied in the $\Lambda \mathrm{XCDM}$ model, although only in a narrow range of the parameter space. In fact, from the definition of the parameter $b$ in (76), the expectation that $\left|\omega_{X}\right|=\mathcal{O}(1)$, and neglecting $\epsilon$, we see that (87) is approximately equivalent to

$$
\Omega_{X}^{0} \gtrsim \frac{\nu \Omega_{M}^{0}}{\omega_{X}}=\mathcal{O}(-\nu) .
$$

Given the fact that $\nu$ was found to be positive and small (cf. Figs. 1 and 2) and, at the same time, $\Omega_{X}^{0}<0$ (see previous section), the above condition does not leave much freedom within the allowed parameter space, roughly $-\nu \leqq \Omega_{X}^{0}<0$. This narrow strip is, however, not necessarily negligible; e.g. if we take $\nu$ of order of $\nu_{0} \sim 10^{-2}$ [cf. Eq. (69)], this possibility is still permitted in the parameter space, see Figs. 1 and 2. In such a case, the present cosmon density could still be of the order or larger (in absolute value) than, say, the current neutrino contribution to the energy density of the Universe $\left(\Omega_{\nu}^{0} \sim 10^{-3}\right)$. No matter how tiny (in absolute value) a negative cosmon contribution to the energy density is, it suffices to take care of the cosmic coincidence problem along the lines that we have explained. Therefore, the adiabatic contribution is perfectly tenable, but the numerical analysis of the subsequent sections remains essentially the same (as we have checked) independently of whether the sound speed of the DE medium is adiabatic or not. For this reason, in what follows we will assume the more general situation of nonadiabatic perturbations, with the understanding that adiabatic ones can do a similar job in the corresponding region of the parameter space.

\section{THE MATTER POWER SPECTRUM}

In this section, we compare the matter power spectrum predicted by the $\Lambda$ XCDM model with the observed galaxy power spectrum measured by the 2dFGRS survey [4]. The $\Lambda \mathrm{XCDM}$ matter power spectrum is found by evolving the perturbation equations (43)-(46) from $a=a_{i}$ to the present $\left(a_{0}=1\right)$, where $a_{i} \ll 1$ is the scale factor at some early time, but well after recombination. In these equations, we must of course use the expansion rate (38) with the full DE density (79).

In order to set the initial conditions at $a=a_{i}$, we use the prediction from the standard $\Lambda \mathrm{CDM}$ model. Indeed, the standard $\Lambda$ CDM model provides a good analytical fit to the $2 \mathrm{dFGRS}$ observed galaxy power spectrum. Taking this fit as our starting point, we compute analytically the values of the $\Lambda \mathrm{CDM}$ perturbations at an arbitrary scale factor. Since the DE does not play an important role until very recently, we may assume that the initial matter and metric perturbations at $a=a_{i}$ for the $\Lambda \mathrm{XCDM}$ model are the same as for the $\Lambda$ CDM model.

\section{A. Initial matter and metric perturbations}

As previously commented, the perturbed equations are evolved from some $a_{i} \ll 1$ to $a_{0}=1$. The value of $a_{i}$ is unimportant, provided that it lies well after recombination (to insure that all the processes encoded in the transfer function have already taken place). For definiteness, we take $a_{i}=1 / 500$, i.e. cosmological redshift $z \sim 500$. Next we specify the initial conditions at $a=a_{i}$.

For the matter and metric perturbations, the initial conditions in the standard cosmological model can be computed analytically $[35,36]$. The matter perturbed equations in the standard $\Lambda \mathrm{CDM}$ model are (43) and (50) taking $\delta_{D}(a)=\theta_{D}(a)=0$. In these conditions, the r.h.s. of the differential equation (50) vanishes and we are left with a homogeneous equation. In the standard model, this equation is just Eq. (51) with $w_{e}=-1$, i.e.

$$
\delta_{M}^{\prime \prime}(a)+\frac{3}{2}\left(2-\tilde{\Omega}_{M}(a)\right) \frac{\delta_{M}^{\prime}(a)}{a}-\frac{3}{2} \tilde{\Omega}_{M}(a) \frac{\delta_{M}(a)}{a^{2}}=0 .
$$

Since matter is conserved, we have $\tilde{\Omega}_{M}(a)=$ $\left(H_{0} / H(a)\right)^{2} \Omega_{M}^{0} / a^{3}$. Moreover, from Eq. (48) (again with $\left.w_{e}=-1\right)$ one can also see that $\tilde{\Omega}_{M}(a)=$ $-(2 a / 3) H^{\prime}(a) / H(a)$. Hence, the homogeneous equation (89) can be conveniently cast as follows:

$$
\delta_{M}^{\prime \prime}(a)+\left(\frac{3}{a}+\frac{H^{\prime}(a)}{H(a)}\right) \delta_{M}^{\prime}(a)-\frac{3}{2} \Omega_{M}^{0} \frac{H_{0}^{2}}{H^{2}(a)} \frac{\delta_{M}(a)}{a^{5}}=0 .
$$

This equation is now in a standard form and can be solved analytically [53]. Let us first introduce the variable $D(a)=$ $\delta_{M}(a) / \delta_{\text {ref }}$ (the so-called growth factor), where $\delta_{\text {ref }}$ is the matter density contrast at some initial scale factor. In the initial matter era $\left(a=a_{i} \ll 1\right)$ wherein the cosmological term is negligible, we have $H^{2}(a) / H_{0}^{2}=\Omega_{M}^{0} a^{-3}$ in very good approximation; then, by imposing the boundary conditions $D(a) \propto a$ and $D^{\prime}(a)=1$, the growing solution of (90) is simply $D(a)=a$, as can be easily checked. In the general case, one can find the solution for the growing mode which reduces to the previous one deep into the matter-dominated era. The final result reads

$$
D(a)=\frac{\delta_{M}(a)}{\delta_{\text {ref }}}=\frac{5 \Omega_{M}^{0}}{2} \frac{H(a)}{H_{0}} \int_{0}^{a} \frac{d \tilde{a}}{\left(\tilde{a} H(\tilde{a}) / H_{0}\right)^{3}} .
$$

In particular, for $H(a) / H_{0}=\left(\Omega_{M}^{0}\right)^{1 / 2} a^{-3 / 2}$ it just boils down to the solution $D(a)=a$ corresponding to the early matter-dominated epoch, as expected. 
Furthermore, Eq. (43) leads to

$$
\hat{h}(a)=2 a H(a) \frac{D^{\prime}(a)}{D(a)} \delta_{M}(a),
$$

and for $D(a)=a$ it renders the initial condition

$$
\hat{h}\left(a_{i}\right)=2 H\left(a_{i}\right) \delta_{M}\left(a_{i}\right)
$$

for the metric fluctuation. Later on, when the DE (i.e. $\rho_{\Lambda}^{0}>$ 0 in the $\Lambda \mathrm{CDM}$ ) starts to play a role, the matter (and metric) fluctuations become suppressed. The suppression is given by the value of the growth factor $D(a)$, which is no longer proportional to the scale factor. ${ }^{11}$ From (91) it is clear that $\delta_{M}(a) / D(a)$ is a constant, which can be written as $\delta_{M}\left(a_{i}\right) / a_{i}$ at early times (when $D\left(a_{i}\right)=a_{i}$ ) and as $\delta_{M}\left(a_{0}\right) / D\left(a_{0}\right)$ at the present time. Therefore,

$$
\begin{gathered}
\delta_{M}\left(a_{i}\right)=\frac{a_{i}}{D_{0}} \delta_{M}\left(a_{0}\right), \\
\hat{h}\left(a_{i}\right)=2 H_{\Lambda}\left(a_{i}\right) \frac{a_{i}}{D_{0}} \delta_{M}\left(a_{0}\right),
\end{gathered}
$$

where $D_{0} \equiv D\left(a_{0}\right)$ and the subindex in $H_{\Lambda}\left(a_{i}\right)$ has been added to emphasize that the initial value of the Hubble parameter is to be computed within the $\Lambda \mathrm{CDM}$ model. Note that, instead of setting the value of $\hat{h}\left(a_{i}\right)$, we could have chosen to put initial conditions on the derivative of the density contrast, $\delta_{M}^{\prime}$. In that case, as it is evident from (94), we would have that $\delta_{M}^{\prime}\left(a_{i}\right)=\delta_{M}\left(a_{0}\right) / D_{0}$. Then the initial value of the metric fluctuation is constrained by Eq. (43) to be $\hat{h}\left(a_{i}\right)=2 H_{\Lambda \mathrm{X}}\left(a_{i}\right) a_{i} \delta_{M}\left(a_{0}\right) / D_{0}$, where now $H_{\Lambda \mathrm{X}}\left(a_{i}\right)$ is the $\Lambda \mathrm{XCDM}$ value of the Hubble function. Note that this value of $\hat{h}\left(a_{i}\right)$ is not exactly the same as that in (95), since $H_{\Lambda}\left(a_{i}\right)$ and $H_{\Lambda \mathrm{X}}\left(a_{i}\right)$ are not identical. However, being that the difference is rather small (as we have checked numerically), the behavior of the perturbations does not depend significantly on that choice.

The Eqs. (94) and (95) give us the initial conditions at $a_{i}=1 / 500$ for the matter and metric perturbations in terms of the density contrast today $\delta_{M}\left(a_{0}\right)$. We associate the latter with the 2dFGRS observed galaxy power spectrum fitted in the $\Lambda \mathrm{CDM}$ model, as detailed below.

The matter power spectrum of the $\Lambda$ CDM model can be approximated as [41]:

$$
P_{\Lambda}(k) \equiv\left|\delta_{M}(k)\right|^{2}=A k T^{2}(k) \frac{g^{2}\left(\Omega_{T}^{0}\right)}{g^{2}\left(\Omega_{M}^{0}\right)},
$$

where $\Omega_{T}^{0}=\Omega_{M}^{0}+\Omega_{\Lambda}^{0}$. It assumes a scale-invariant

\footnotetext{
${ }^{11}$ Notice that if $\tilde{\Omega}_{\Lambda}$ is small and essentially constant, then the growth factor $D(a)$ takes the approximate form $D(a) \sim a^{n}$, with $n=1-6 \tilde{\Omega}_{\Lambda} / 5<1$, as it follows from (52) for $w_{e}=-1$, or from (89). This demonstrates, if only roughly, the suppression behavior in an explicit analytic way. In the general $\Lambda \mathrm{CDM}$ case, however, the solution for the growing mode is given by (91), in which $H$ is the full expansion rate of the standard model.
}

(Harrison-Zeldovich) primordial spectrum, as generically predicted by inflation. This primordial spectrum is modified when taking into account the physical properties of different constituents of the Universe, in particular, the interactions between them. All these effects are encoded into the scale-dependent transfer function $T(k)$, which describes the evolution of the perturbations through the epochs of horizon crossing and radiation/matter transition. The growth at late times which, in the $\Lambda$ CDM model, is independent of the wave number, is described by the growth function $g(\Omega)$. Finally, $A$ is a normalization factor.

The transfer function can be accurately computed by solving the coupled system formed by the Einstein and the Boltzmann equations. Although a variety of numerical fits have been proposed in the literature, here we use the socalled BBKS transfer function [54]:

$$
\begin{aligned}
T(k)= & \frac{\ln (1+2.34 q)}{2.34 q}\left[1+3.89 q+(16.1 q)^{2}+(5.46 q)^{3}\right. \\
& \left.+(6.71 q)^{4}\right]^{-1 / 4}
\end{aligned}
$$

where

$$
q=q(k) \equiv \frac{k}{(h \Gamma) \mathrm{Mpc}^{-1}}
$$

and $\Gamma$ is the Sugiyama's shape parameter $[35,55]$

$$
\Gamma \equiv \Omega_{M}^{0} h e^{-\Omega_{B}^{0}-\sqrt{h / 0.5}\left(\Omega_{B}^{0} / \Omega_{M}^{0}\right)} .
$$

On the other hand, for the growth function we assume the following approximation [56]:

$$
g(\Omega)=\frac{5 \Omega}{2}\left[\Omega^{4 / 7}-\Omega_{\Lambda}^{0}+\left(1+\frac{\Omega}{2}\right)\left(1+\frac{\Omega_{\Lambda}^{0}}{70}\right)\right]^{-1},
$$

which reflects the suppression in the growth of perturbations caused by a positive cosmological constant. The normalization coefficient $A$ is related to the CMB anisotropies through [41]

$$
A=\left(2 l_{H}\right)^{4} \frac{6 \pi^{2}}{5} \frac{Q_{\text {rms-PS }}^{2}}{T_{0}^{2}},
$$

where $Q_{\text {rms-Ps }}$ is the quadrupole amplitude of the CMB anisotropy (see below for more detailed explanations), $l_{H} \equiv H_{0}^{-1} \simeq 3000 h^{-1} \mathrm{Mpc}$ is the Hubble radius, and $T_{0} \simeq$ $2.725 \mathrm{~K}$, the present $\mathrm{CMB}$ temperature. Therefore, the value of the normalization factor $A$ could in principle be inferred from measurements of the CMB. However, we have obtained it by fitting the power spectrum (96) to the 2dFGRS observed galaxy power spectrum [4], as discussed below.

We assume $h=0.7$ for the reduced Hubble parameter and a spatially flat universe with $\Omega_{M}^{0}=0.3$ (hence $\Omega_{\Lambda}^{0}=$ 0.7 for the flat $\Lambda \mathrm{CDM}$ model) in order to be consistent with our assumption in previous analyses [17,23]. The fit to the 
2dFGRS observed galaxy power spectrum $P_{2 \mathrm{dF}}(k)$ is obtained assuming the matter budget composed of a baryonic part $\Omega_{B}^{0}=0.04$ and a dark matter contribution $\Omega_{\mathrm{DM}}^{0}=$ 0.26 . In order to calculate the best fit, we use the formula (96) to minimize the $\chi$-square distribution

$$
\chi^{2} \equiv \frac{1}{n_{\mathrm{dof}}} \sum_{k} \frac{\left[P_{2 \mathrm{dF}}(k)-P_{\Lambda}(k)\right]^{2}}{\sigma^{2}(k)}
$$

in terms of the normalization $A$. There are 39 values of $k$ in the 2dFGRS data, so the number of degrees of freedom is $n_{\text {dof }}=38$. We find, as best fit, the value

$$
A=8.99 \times 10^{5} h^{-4} \mathrm{Mpc}^{4}
$$

with $\chi^{2}=0.43$. From (101) we see that this value of $A$ implies $Q_{\text {rms-PS }} \simeq 20.85 \mu \mathrm{K}$. Let us now clarify that $Q_{\text {rms-PS }}$ is not the observed quadrupole CMB anisotropy (usually denoted $Q_{\text {rms }}$ ), but rather the value derived from a fit to the entire CMB power spectrum (PS). For a powerlaw spectrum with $n=1$ (i.e. for a scale-invariant PS), the COBE team obtained $Q_{\text {rms-PS }}=18 \pm 1.6 \mu \mathrm{K}$ [57], and moreover they found that the observed $Q_{\mathrm{rms}}$ is smaller

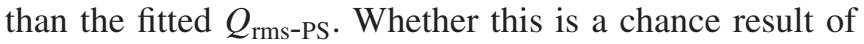
cosmic variance or reflects the physical cosmology is not known [57]. Our fitted value for $Q_{\text {rms-PS }}$ falls within the $2 \sigma$ range of the corresponding $\mathrm{COBE}$ value (although when the quadrupole itself is not used in the fit, the COBE uncertainties become larger [57]). As several authors have noted $[55,58]$, such a normalization may be inadequate for models with a cosmological constant, given the fact that the CMB spectra of $\Lambda$-dominated models is quite different from a simple power law, especially at large scales (low multipoles). For instance, in [58] an alternative normalization for the $\Lambda \mathrm{CDM}$ model is proposed, which for $h=0.8$ and $\Omega_{\Lambda}^{0}=0.7$ yields $Q_{\text {rms-PS }}=22.04 \mu \mathrm{K}$, with an error of the order of $11 \%$, which is in agreement with our result. In general one can find a number of different values for $Q_{\text {rms-PS }}$ in the literature depending on the kind of analysis performed or the data set used, and this is why we preferred to compute the normalization directly from a fit to the matter power spectrum. Finally, let us emphasize that our value for $Q_{\text {rms-PS }}$ lies within the $95 \%$ confidence interval for the observed quadrupole anisotropy $\left(Q_{\mathrm{rms}}\right)$ by both COBE [57] and WMAP [59].

Therefore, we will assume (94) and (95) as the initial conditions for the matter and metric perturbations, identifying $\delta_{M}\left(a_{0}\right)$ with $\delta_{M}(k)$ from the formula (96) using the fitted coefficient (103).

\section{B. The $\triangle \mathrm{XCDM}$ matter power spectrum}

The procedure discussed above helps us to set initial conditions for the matter and metric perturbations in the $\Lambda \mathrm{XCDM}$ model. However, since the $\Lambda \mathrm{CDM}$ model does not include DE perturbations, we should set independent initial conditions on $\delta_{D}\left(a_{i}\right)$ and $\theta_{D}\left(a_{i}\right)$. As already dis- cussed, the scales relevant to the matter power spectrum remain always well below the sound horizon (65) and we expect negligible DE perturbations at any time. Thus, the most natural choice for the initial values of the DE perturbations is

$$
\delta_{D}\left(a_{i}\right)=0, \quad \theta_{D}\left(a_{i}\right)=0 .
$$

Indeed, this is not the only reasonable choice. For instance, we could have also assumed the adiabatic initial condition (54) for the DE density contrast, i.e. $\delta_{D}\left(a_{i}\right)=$ $\left(1+w_{e}\left(a_{i}\right)\right) \delta_{M}\left(a_{i}\right)$, with $\delta_{M}\left(a_{i}\right)$ given by Eq. (94). Again, it has been checked that the evolution of the perturbations does not depend significantly on the particular initial condition used.

Assuming the initial conditions (94), (95), and (104) for the matter, metric, and DE perturbations at $a_{i}$, respectively, we can solve the perturbed equations (43)-(46). Equivalently, we can solve (44) and (45) to obtain $\left(\delta_{D}, \theta_{D}\right)$ and then (50) to get the matter density fluctuations today $\delta_{M}(k, a=1)$ for any dark energy model. In particular, we can (as a consistency check) solve the perturbation equations for the $\Lambda \mathrm{CDM}$ model [in that case $\delta_{D}(a)=$ $\theta_{D}(a)=0$, so the only equations needed are (43) and (46)]. In doing so, we recover exactly the spectrum $P_{\Lambda}$ defined in (96).

Now we proceed to compute the spectrum of the $\Lambda \mathrm{XCDM}, P_{\Lambda \mathrm{X}}(k)$. In order to better compare the shape of the different spectra and the goodness of their fit to the 2dFGRS observed galaxy power spectrum, we will normalize them at the smallest length scale considered $\ell \sim$ $k^{-1}$, i.e. at $k=0.2$, taking the $\Lambda \mathrm{CDM}$ spectrum (96) as reference. To this purpose, we introduce a normalization factor $A_{\Lambda \mathrm{X}}$ in the matter power spectrum:

$$
P_{\Lambda \mathrm{X}}(k) \equiv A_{\Lambda \mathrm{X}}\left|\delta_{M}(k, a=1)\right|^{2} .
$$

Notice that the normalization factor $A_{\Lambda \mathrm{x}}$ gives us the difference in the matter power spectrum of the model with respect to that of the $\Lambda \mathrm{CDM}$ at the specific scale $k=0.2$. Let us clarify that the reason for choosing this scale for the normalization is that, as discussed in Sec. V, the smaller the length scale (i.e. the higher the value of $k$ ) the less important the DE perturbations are. Therefore, at $k=0.2$, the matter power spectrum of the model should not depend significantly on whether we consider the effect of the DE perturbations; in particular, it should be independent of the speed of sound $c_{s}$. For larger scales, however, the DE perturbations can be more significant and, as we shall see below, they may introduce some differences in the shape of the power spectrum, which are nevertheless small in the linear regime.

The $\Lambda$ XCDM power spectrum was calculated for two fiducial values of the DE speed of sound, $c_{s}^{2}=1$ and $c_{s}^{2}=$ 0.1 and several combinations of the parameters $\nu, \omega_{X}$, and $\Omega_{\Lambda}^{0}$. For values of the parameters allowed in Figs. 1 and 2 

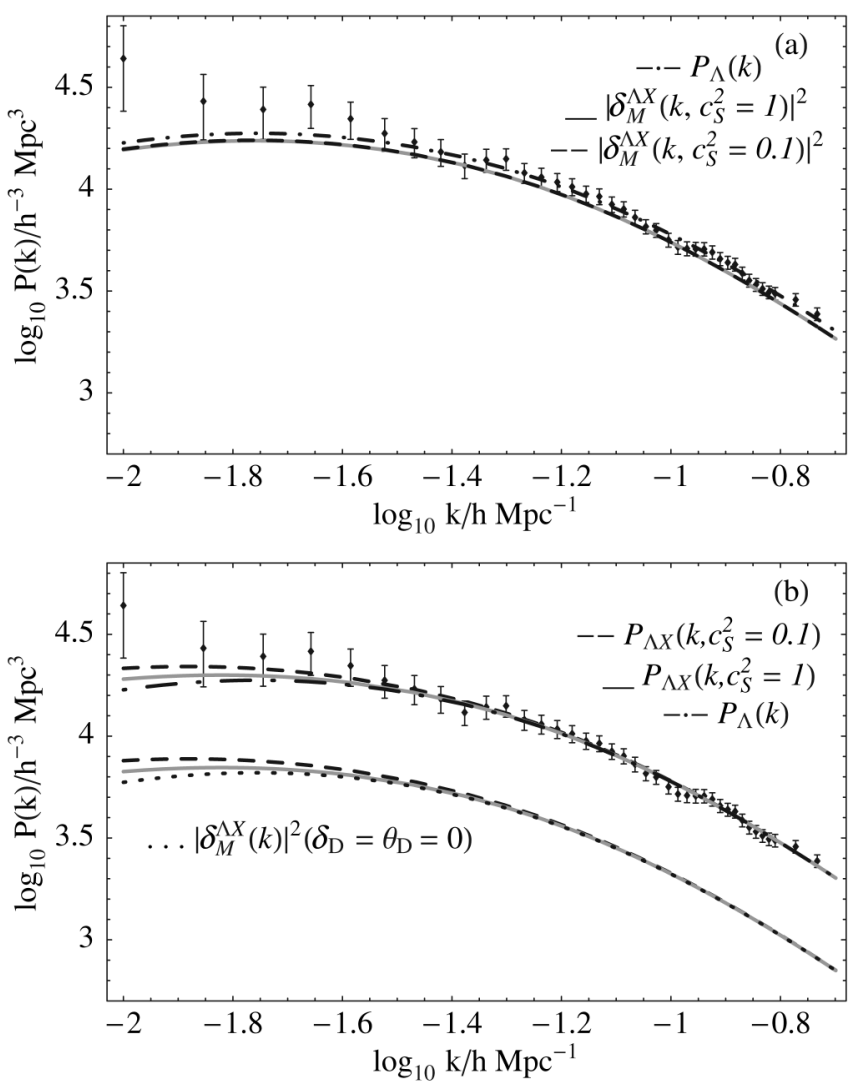

FIG. 3. The $\Lambda \mathrm{CDM}$ power spectrum $P_{\Lambda}(k)$ (dot-dashed line) versus the normalized and non-normalized spectrum predicted by the $\Lambda \mathrm{XCDM}$ model, for DE sound speeds $c_{s}^{2}=0.1$ (dashed line) and $c_{s}^{2}=1$ (solid/gray line): (a) for a set of parameters allowed by the analysis of Ref. [23] (cf. shaded and striped region in our Figs. 1 and 2), $\Omega_{\Lambda}^{0}=0.8, \nu=\nu_{0} \equiv 2.6 \times 10^{-2}$, and $w_{X}=-1.6$. The corresponding curves $P_{\Lambda}(k)$ and $P_{\Lambda \mathrm{X}}(k)$ coincide in this case; (b) for a set of parameters not allowed by the F test [23] (points in the striped, but nonshaded, region in our Fig. 1), $\Omega_{\Lambda}^{0}=+0.35, \nu=-0.2$, and $w_{X}=-0.6$. In this case, $P_{\Lambda \mathrm{X}}(k)$ presents a slight deviation as compared to $P_{\Lambda}(k)$ at large scales (i.e. at small $k$ ). The lower set of curves in (b) displays the real (non-normalized) growth, see the text.

(shaded and striped region) we find that $A_{\Lambda \mathrm{X}} \approx 1$ (within $\sim 10 \%$ of accuracy).

In Fig. 3(a), we put together the 2dFGRS observed galaxy power spectrum, the $\Lambda \mathrm{CDM}$ spectrum, and the normalized and unnormalized $\Lambda \mathrm{XCDM}$ one, for the set of parameters $\Omega_{\Lambda}^{0}=0.8, \nu=\nu_{0} \equiv 2.6 \times 10^{-2}$, and $w_{X}=$ -1.6 , which are allowed in Figs. 1 and 2. For these values, we have obtained a normalization factor $A_{\Lambda X} \cong 1.1$ and an accurate agreement between the $\Lambda \mathrm{XCDM}$ power spectrum and $P_{\Lambda}(k)$. This was expected since we are assuming allowed values of the parameters, i.e., values already consistent with LSS data according to the "effective" approach used in [23] (cf. the discussion in Sec. VII). Therefore, the predicted power spectrum from the $\Lambda \mathrm{XCDM}$ ought to be very close to the $\Lambda \mathrm{CDM}$ one, which is in fact what we have substantiated now by explicit numerical check.

However, for values of the parameters out of the allowed region in Fig. 1 the predicted matter power spectrum can differ significantly from the $\Lambda \mathrm{CDM}$ one, $P_{\Lambda}(k)$. This occurs mainly for points that do not satisfy the F-test condition [23], even if the other observable constraints [namely the ones related to nucleosynthesis and the present value of the EOS (cf. Sec. VII)] are fulfilled. Let us remind that the $\mathrm{F}$ test consists in requiring that the matter power spectrum of the model under consideration (in this case, the $\Lambda \mathrm{XCDM}$ model) differs from that of the $\Lambda \mathrm{CDM}$ in less than $10 \%$, under the assumption that DE perturbations can be neglected. Given the fact (explicitly analyzed here) that the DE perturbations should not play a very important role, it is reasonable to expect that the F test should be approximately valid even when we do not neglect the DE perturbations. Thus, the $\Lambda \mathrm{XCDM}$ model should exhibit a large deviation in the amount of growth with respect to the $\Lambda \mathrm{CDM}$ precisely for those points failing the $\mathrm{F}$ test. Points of this sort are those located in the striped region, but outside the shaded one in Fig. 1. For these points, we should expect an anomalously large normalization factor $A_{\Lambda X}$ (namely, the factor that controls the matching of the two overall shapes) and, at the same time, we may also observe an evident scale dependence in the power spectrum, i.e. some significant difference in the predicted shape as compared to the $\Lambda \mathrm{CDM}$ one. Such potentially relevant scale dependence (or $k$ dependence) is introduced by the DE perturbations themselves through the last term on the r.h.s of (39) and is eventually fed into Eqs. (44)-(46).

As a concrete example, let us consider Fig. 3(b) where we compare the $2 \mathrm{dFGRS}$ observed galaxy power spectrum and $P_{\Lambda}(k)$ with the $\Lambda$ XCDM matter power spectrum $P_{\Lambda \mathrm{X}}(k)$ for the following set of parameters: $\Omega_{\Lambda}^{0}=$ $+0.35, \nu=-0.2$, and $w_{X}=-0.6$. These values fulfill the nucleosynthesis bound (constraint No. 1 in Sec. VII), specifically, we have $|\epsilon|=0.08$ for these parameters (meaning that DE density at the nucleosynthesis time represents roughly only $8 \%$ of the total energy density); and satisfy also the current EOS constraint (No. 3 in Sec. VII): $w_{e}^{0}=-0.8$. However, this choice of parameters largely fails to satisfy the constraint No. 4, i.e. the F test: indeed, we find $F=2.06$, which implies that the discrepancy in the amount of growth with respect to the $\Lambda \mathrm{CDM}$ when we neglect DE perturbations is more than $200 \%$. As expected, for such set of parameters we encounter a large normalization factor for the two fiducial DE sound speeds $c_{s}^{2}=1$ and $c_{s}^{2}=0.1$ that we are using in our analysis (on average $A_{\Lambda \mathrm{X}} \simeq 2.7$ ). This is reflected in the evident gap existing between the upper and lower set of curves in Fig. 3(b). The lower set reflects the real growth $\left|\delta_{M}(k)\right|^{2}$ of matter perturbations before applying the normalization factor. Such a normalization consists in the following: for the smallest scale available in the data, the $\Lambda \mathrm{XCDM}$ curves 
have been shifted upwards until they match up with the standard $\Lambda \mathrm{CDM}$ prediction. Apart from the overall gap between the two sets of curves, we also find a significant shape deviation with respect to the standard $\Lambda$ CDM model at large scales, as can be seen in Fig. 3(b). The smaller the speed of sound, the greater the deviation, as discussed in more depth in the next section.

\section{MATTER AND DARK ENERGY DENSITY FLUCTUATIONS}

As we have discussed in Sec. V, the DE fluctuations $\delta_{D}$ should oscillate and become eventually negligible as compared to the matter fluctuations $\delta_{M}$, especially at small scales (inside the sound horizon). However, as also noted above, for values that significantly violate the $F$ test [23], the power spectrum and its shape can be noticeably different from that of the $\Lambda \mathrm{CDM}$ model (cf. Fig. 3(b)). This suggests that, under such circumstances, the DE density perturbations are not completely negligible owing to the fact that the term which depends on $k$ in the perturbation equations is also proportional to $\delta_{D}$-see Eq. (45). In addition, there appears a suppression of the growth of matter fluctuations in comparison with the growth predicted by the $\Lambda \mathrm{CDM}$ model. This inhibition of matter growth is characteristic of cosmologies where the DE behaves quintessencelike, i.e. when the DE density decreases with the expansion, whereas phantomlike DE (increasing with the expansion) would cause the opposite effect (an enhancement of the power). A similar situation was also observed in Ref. [41] for models with pure running $\Lambda$, where in the case $\nu>0$ (in which $\Lambda$ decreases with the expansion) there is an inhibition of growth while for $\nu<0$ (when $\Lambda$ increases with the expansion) there is an enhancement—see also [60-62] for other studies.

Let us clarify that these differences in the amount of growth are present even if we neglect the DE perturbations, see [23]. In fact, the effect of the latter is very small, specially for allowed values of the parameters, and becomes noticeable only at large scales. At these scales, we find that the DE perturbations tend to compensate the suppression produced at the background level. This slight enhancement is greater the smaller the DE sound speed is. Such a feature can be appreciated in Fig. 4, where we compare the growth of the matter fluctuations at a large scale $\ell \sim k^{-1}$ (with $k=0.01$ ) predicted by both the $\Lambda \mathrm{CDM}$ and $\Lambda \mathrm{XCDM}$ models for the two fiducial sound speeds of the DE considered before and for the same values of the parameters as in Fig. 3.

The growth of matter density fluctuations for the $\Lambda \mathrm{XCDM}$ model is in agreement with the predicted one by the $\Lambda$ CDM model (the dot-dashed and black line) in Fig. 4(a), for the set of parameters in the allowed region, whereas in Fig. 4(b) we see the previously commented suppression for the set of parameters not satisfying the $\mathrm{F}$ test. The former case can be compared with Fig. 5(a) in
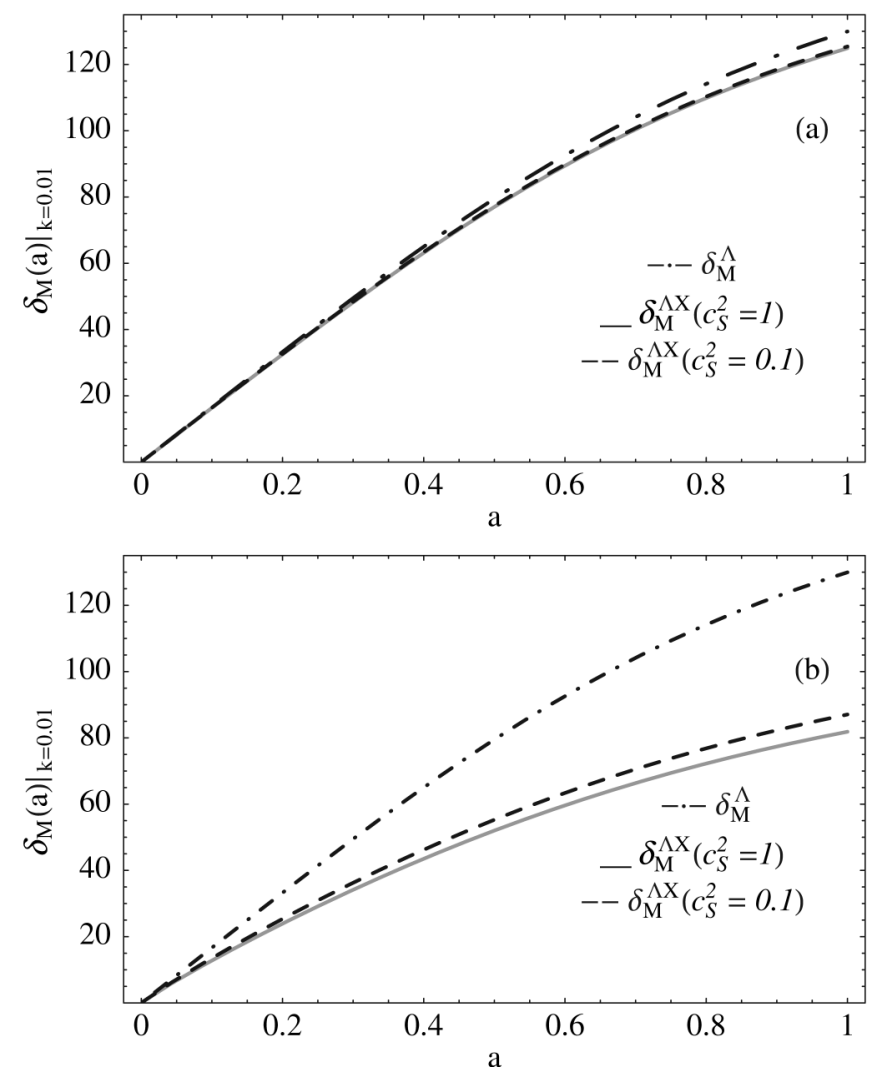

FIG. 4. The $\Lambda \mathrm{XCDM}$ matter density fluctuations at a fixed large scale $k=0.01$ (in units of $h \mathrm{Mpc}^{-1}$ ) as a function of the scale factor $a$ in comparison with those predicted by the $\Lambda \mathrm{CDM}$ model (dot-dashed line). For the former we have assumed the same values of the parameters and meaning of the lines as in Fig. 3: (a) for the set of parameters in the allowed region; (b) for the set of parameters not allowed by the $\mathrm{F}$ test in [23].

which we have assumed the same set of allowed parameters; as expected, we find completely negligible DE fluctuations today and in the recent past, in agreement with the F-test assumption [23] which means completely negligible DE fluctuations at large scales and a maximum $10 \%$ of deviation from the $\Lambda \mathrm{CDM}$ growth of matter density fluctuations. On the other hand, values of the parameters not satisfying the $F$ test present not only suppression on the growth of matter density fluctuations, as shown in Fig. 4(b), but also larger DE fluctuations today and in the recent past, as shown in Fig. 5(b).

Furthermore, as discussed in Sec. V, the growth of DE fluctuations is expected to oscillate at small scales and rapidly decay, the fact that legitimates our assumptions for the initial conditions of the DE perturbations. We show these oscillations for an allowed set of parameters in Fig. 6. Similar behavior is obtained for values of the parameters not allowed by the F test and for both DE sound speeds $c_{s}^{2}=1$ and $c_{s}^{2}=0.1$. The amplitude of the DE growth starts negligible $\left(\sim 10^{-3}\right)$ and rapidly decays to zero, as shown in Fig. 6. 

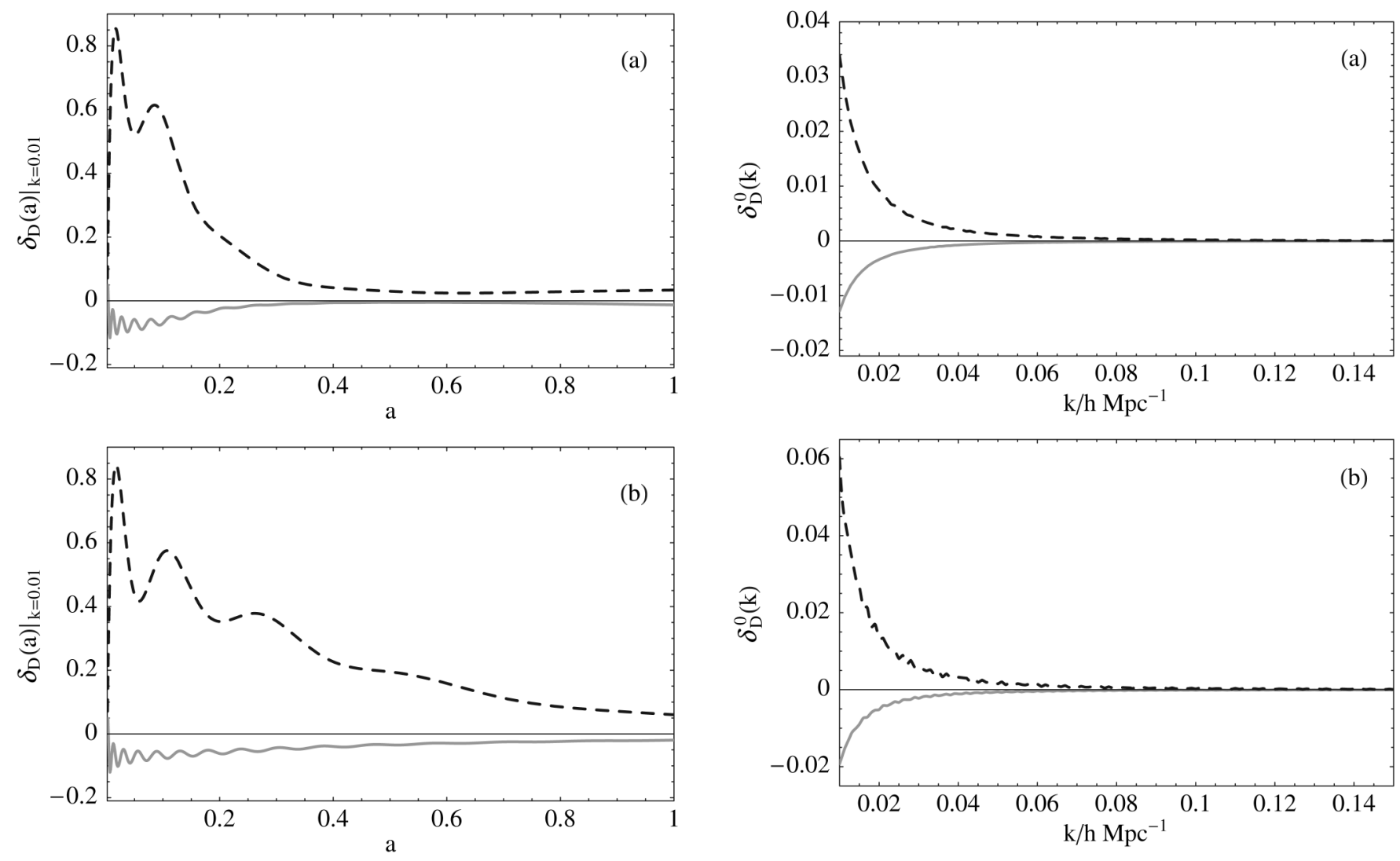

FIG. 5. The $\Lambda \mathrm{XCDM}$ growth of DE fluctuations at the large scale $k=0.01$ (in units of $h \mathrm{Mpc}^{-1}$ ) as a function of the scale factor $a$. We have assumed the same values of the parameters as in Fig. 3 for DE sound speed $c_{s}^{2}=0.1$ (dashed line) and $c_{s}^{2}=1$ (solid/gray line): (a) for the set of parameters in the allowed region; (b) for the set of parameters not allowed by the $\mathrm{F}$ test in [23].

In Fig. 7 we plot the present value of the DE perturbations as a function of the wave number for the two sets of allowed (Fig. 7(a)) and nonallowed (Fig. 7(b)) parameters used in the previous plots. We see that the DE perturbations

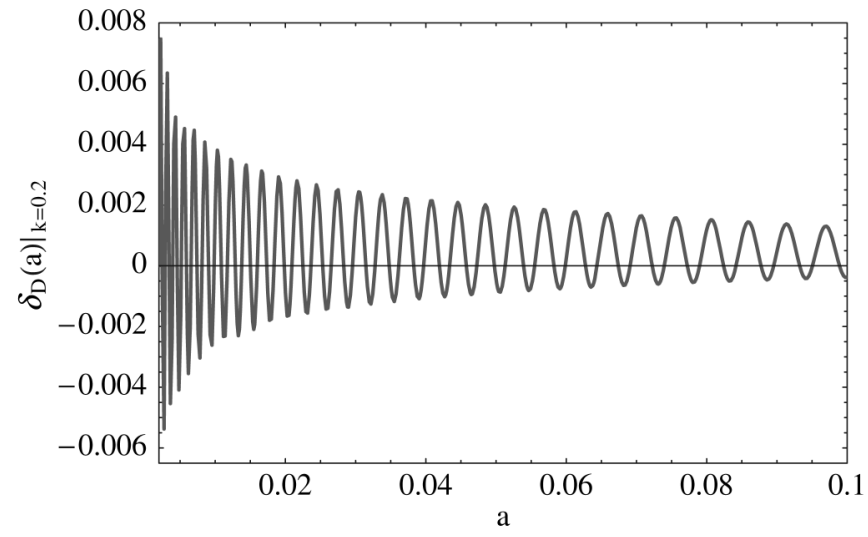

FIG. 6. The $\Lambda \mathrm{XCDM}$ growth of $\mathrm{DE}$ fluctuations for a small scale $k=0.2$ (in units of $h \mathrm{Mpc}^{-1}$ ) and the same set of allowed parameters assumed before, and for DE sound speed $c_{s}^{2}=0.1$.

FIG. 7. The scale dependence of DE fluctuations today $(a=1)$ for the same values of parameters and meaning of the lines as in Fig. 5. They rapidly decay at small scales (large $k$ ).

are negligible at small scales (large $k$ ), whereas they become larger at larger scales. This is because by increasing the scale we are getting closer to the sound horizon, as discussed in Sec. V. We also see that the DE perturbations are larger for the parameters not allowed by the F test, which explains why the shape of the matter power spectrum differs from that of the $\Lambda \mathrm{CDM}$ in this case (cf. Fig. 3(b)]. However, when comparing with Fig. 4, we see that even at the largest explored scale $(k=0.01)$ the ratio $\delta_{D} / \delta_{M}$ remains rather small, staying at the level of $10^{-3}$. Finally, let us comment that the DE density contrast can become negative with the evolution, as in this case happens for $c_{s}^{2}=1$.

As discussed in Sec. V, the decay of the DE perturbations takes place once the term proportional to $k^{2}$ in (45) becomes dominant. This same term is also responsible for the exponential growth of the (DE) perturbations in the adiabatic case or, more generally, whenever $c_{s}^{2}$ is negative. In order to better appreciate its influence, it is useful to compare the evolution of the DE perturbations in the adiabatic case (for the most common situation where $c_{a}^{2}<$ $0)$ and the nonadiabatic one $\left(c_{s}^{2}>0\right)$ with the scenario in which $c_{s}^{2}=0$, since in the latter the term proportional to $k^{2}$ disappears from the equations. This is precisely what has been done in Fig. 8(a) for the allowed set of parameters 


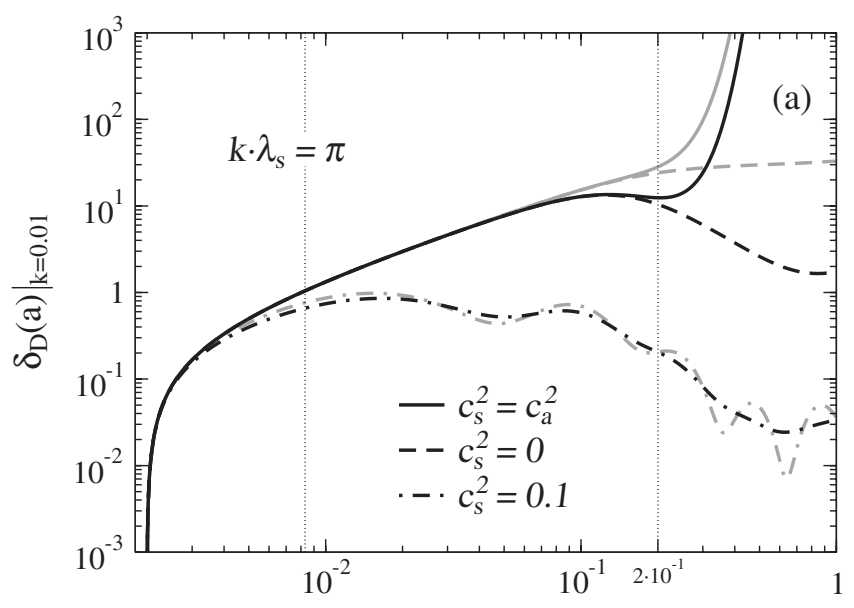

a

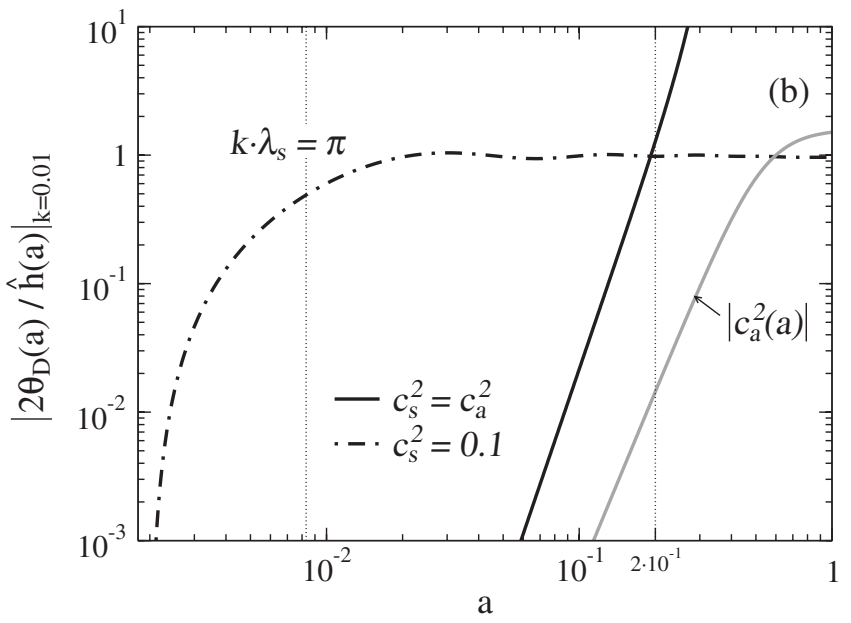

FIG. 8. (a) Evolution of the DE perturbations for the same set of parameters as in Fig. 3(a), at the large scale $k=0.01$ and for three different speeds of sound: $c_{s}^{2}=c_{a}^{2}<0$ (solid line), $c_{s}^{2}=0$ (dashed line), and $c_{s}^{2}=0.1$ (dot-dashed line). For the latter, the DE perturbations begin to decay after the sound horizon crossing (characterized by the condition $k \lambda_{s}=\pi$ ), whereas in the adiabatic case $\delta_{D}$ starts to grow exponentially at $a \simeq 0.2$. The last term in Eq. (44) may be neglected (gray lines) without altering the qualitative behavior, which is triggered by the $\theta_{D}$-term and, ultimately, by the one proportional to $k^{2}$ in (45); (b) Comparison of the two terms inside the curly brackets in (44) for the $c_{s}^{2}=0.1$ and adiabatic cases. When the $\theta_{D}$-term becomes important, the stabilization (resp. unbounded growth) of the nonadiabatic (resp. adiabatic) perturbations becomes manifest.

used throughout this section. In that figure, the evolution of the DE density contrast at a sufficiently large scale $\ell \sim k^{-1}$ for which the DE perturbations can be sizeable is shown (namely at $k=0.01$ ). We illustrate the effect for three different regimes of the speed of sound: $c_{s}^{2}=c_{a}^{2}$ (with $\left.c_{a}^{2}<0\right), c_{s}^{2}=0$, and $c_{s}^{2}=0.1$. The gray lines represent the evolution of $\delta_{D}$ when the last term in Eq. (44) is neglected, showing indeed that the qualitative behavior of the perturbations in the adiabatic and $c_{s}^{2}=0.1$ cases does not stem from that term.
For $c_{s}^{2}=0.1$, the scale considered is initially (i.e. at $a_{i}=1 / 500$ ) larger than the sound horizon, and thus the term proportional to $k^{2}$ is negligible at the beginning of the evolution. The same is true for the adiabatic case because, in the asymptotic past, the effective EOS of the $\Lambda \mathrm{XCDM}$ model resembles that of matter/radiation $\left(w_{e}(a) \rightarrow w_{m}\right.$ for $a \rightarrow 0)$ [17] and, thus, we have $c_{s}^{2}=c_{a}^{2} \simeq w_{e} \simeq 0$ in the matter-dominated epoch. (We recall that in all our discussion we remain in the matter epoch, equality being at $a \sim$ $10^{-4}$ ). Therefore, the term proportional to $k^{2}$ is initially unimportant in all the three cases and this makes the perturbations to evolve in a nearly identical fashion at these first stages, as can be clearly seen from Fig. 8(a).

As the evolution continues, the curve corresponding to $c_{s}^{2}=0.1$ begin to depart from the others. This occurs mainly from the instant when the sound horizon is crossed, i.e. when the wavelength of the $k$-mode gets comparable to the sound horizon; such an instant can be defined through the condition $k \lambda_{s}=\pi$, similarly as in [43]. Then, the term proportional to $k^{2}$ begins to dominate, which in turn makes the DE velocity gradient $\theta_{D}$ to rapidly increase and the $\mathrm{DE}$ perturbations to decay. Later on, the term proportional to $k^{2}$ becomes important also in the adiabatic case. Because of the different sign $\left(c_{a}^{2}<0\right)$, the effect that it triggers is now opposite to the one observed in the $c_{s}^{2}=0.1$ case; thus, instead of getting stabilized, the DE perturbations initiate an exponential growth.

The previous features can be further assessed in a quantitative way by comparing the numerical importance (in absolute value) of the two terms inside the curly brackets in (44). In Fig. 8(b), we plot the ratio between these two terms for both the adiabatic case (with $c_{a}^{2}<0$ ) and the nonadiabatic situation $\left(c_{s}^{2}=0.1\right)$ (note that the term proportional to $H^{2} / k^{2}$ may be neglected for the sub-Hubble perturbations we are dealing with). Comparison with Fig. 8(a) reveals that it is precisely when the term proportional to $\theta_{D}$ stops being negligible that the evolution of the perturbations begins to depart from the $c_{s}^{2}=0$ case. The absolute value of the adiabatic speed of sound is also shown, in order to illustrate the ultimate reason for the startup of the exponential growth in the adiabatic mode: it is only when $c_{a}^{2}$ begins to depart significantly from 0 that the term proportional to $k^{2}$ becomes important, which in turn triggers a rocket increase of the velocity gradient $\theta_{D}$.

As we have discussed in connection to Fig. 8(a), the initial evolution of the perturbations in the $\Lambda$ XCDM model is nearly the same for any of the three values of the speed of sound. In fact, in the adiabatic case, and given the behavior of the effective EOS in the asymptotic past, the conditions that lead to the simplified setup (53) hold. Therefore, that is the equation initially controlling the evolution of $\delta_{D}$. For $c_{s}^{2}=0$ we arrive at exactly the same equation, whereas for positive $c_{s}^{2}$ the resulting equation only differs by the last term in (44), which, as it has been previously discussed, happens to be negligible at least during the first stages of 
the evolution. Notice that for $w_{e} \simeq$ const., Eq. (53) integrates to $\delta_{D}(a)=\left(1+w_{e}\right) \delta_{M}+C$, where $C$ is a constant determined by the initial conditions. In Sec. IV, we pointed out that $C=0$ corresponds to the adiabatic initial condition (54). However, for the alternate initial condition (104), and taking into account that $w_{e}\left(a_{i}\right) \simeq w_{M}=0$ for the $\Lambda$ XCDM model in the early matter-dominated epoch [17], we have $C=-\delta_{M}\left(a_{i}\right)$ and thus $\delta_{D}(a)=\delta_{M}(a)-$ $\delta_{M}\left(a_{i}\right)$. From here we find that the ratio between DE and matter perturbations in the early times of the evolution reads:

$$
\frac{\delta_{D}(a)}{\delta_{M}(a)}=1-\frac{\delta_{M}\left(a_{i}\right)}{\delta_{M}(a)}
$$

This simple predicted behavior is confirmed from the numerical analysis in Fig. 9, where again the allowed set of parameters has been used. We see that the ratio $\delta_{D} / \delta_{M}$ starts being 0 , and subsequently as the matter perturbations grow the last term in (106) diminishes, until the asymptotic value $\delta_{D} / \delta_{M}=1$ is reached. This value is maintained until the conditions leading to (53) cease to be valid. In the adiabatic and $c_{s}^{2}>0$ cases, this happens when the term proportional to $k^{2}$ on the r.h.s. of Eq. (45) can no longer be neglected. On the other hand, when $c_{s}^{2}=0$, the $\delta_{D} / \delta_{M} \simeq$ 1 regime is abandoned at the point when the effective EOS starts acquiring sizable negative values. Moreover, being the term proportional to $k^{2}$ absent, it is now the last term on the r.h.s. of Eq. (44) - which was irrelevant for the other two cases - the one that tends to stabilize the DE perturbations (see also Fig. 8(a)).

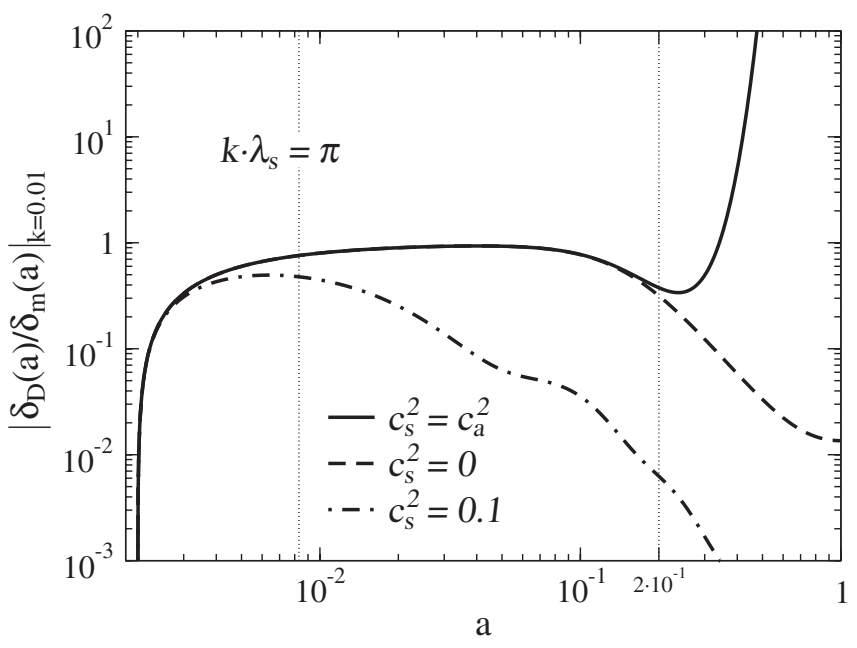

FIG. 9. Evolution of the ratio between DE and matter perturbations for the same parameter values as in Fig. 8. The special regime of perturbations (53) is seen to be approximately realized during the first stages of the evolution, for any of the three considered speeds of sound. Since $w_{e} \rightarrow 0$ for small $a$ in the $\Lambda \mathrm{XCDM}$ model, we expect $\delta_{D} / \delta_{M} \simeq 1$ [cf. Eq. (106)] until the conditions leading to (53) no longer hold. One can clearly confirm this situation in the figure.
From the detailed analysis that we have presented here, we conclude that the approximation of neglecting the DE perturbations can be justified [23]. But this does not mean that the computation of these perturbations is useless. Indeed, the issue at stake here is not so much the quantitative impact of the DE fluctuations upon the matter power spectrum - which is actually negligible, as we have seenbut rather the fact that the DE perturbations may be consistently defined in a certain subregion of the parameter space only. Of course this subregion cannot be detected within the context of the effective approach [23]. Therefore, in general, the computation of the DE perturbations may have a final quantitative bearing on this kind of analysis since it may further restrict the physical region of the parameter space in a very significant way. In short, even though the simultaneous account of the DE perturbations has a small numerical effect on the matter power spectrum within the domain where the full system of cosmological perturbations is well defined, it may nevertheless prove to be a highly efficient method for excluding large regions of parameter space where that system is ill defined. The upshot is that the combined analysis of the DE and matter perturbations may significantly enhance the predictivity of the model, as we have indeed illustrated in detail for the nontrivial case of the $\Lambda \mathrm{XCDM}$ model of the cosmic evolution.

\section{DISCUSSION AND CONCLUSIONS}

In this paper, we have addressed the impact of the cosmological perturbations on the coincidence problem. In contrast to the previous study [23], where this problem was examined in a simplified "effective approach" in which the DE perturbations were neglected, in the present work we have taken them into account in a full-fledged manner. We find that the results of the previous analysis were reasonable because the DE perturbations generally tend to smooth at scales below the sound horizon. However, the inclusion of the DE perturbations proved extremely useful to pin down the physical region of the parameter space and also to put the effective approach within a much larger perspective and to set its limitations.

First of all, we have performed a thorough discussion on the coupled set of matter and DE perturbations for a general multicomponent fluid. This has prepared the ground to treat models in which the DE is a composite medium with a variable EOS. We have concentrated on those cases in which the DE, despite its composite nature, is described by a self-conserved density $\rho_{D}$. Notice that if matter is covariantly conserved, the covariant conservation of the DE is mandatory. In particular, this is the situation for the standard $\Lambda$ CDM model, although in this case the self-conservation of the DE appears through a trivial cosmological constant term, $\rho_{\Lambda}=\rho_{\Lambda}^{0}$, which remains imperturbable throughout the entire history of the Universe. One may nevertheless entertain generalized frameworks where 
the DE is not only self-conserved, but is nontrivial and dynamical. This is not a mere academic exercise; for instance, in quantum field theory in curved space-time we generally expect that the vacuum energy should be a running quantity $[13,15,16]$. Therefore, in such cases, the $\mathrm{CC}$ density becomes an effective parameter that may evolve typically with the expansion rate, $\rho_{\Lambda}=\rho_{\Lambda}(H)$, and constitutes a part of the full (dynamical) DE of the composite cosmological system with variable EOS. In these circumstances, if the gravitational coupling $G$ is constant, the running CC density $\rho_{\Lambda}=\rho_{\Lambda}(H)$ cannot be covariantly conserved unless other terms in the effective action of this system compensate for the $\mathrm{CC}$ variation. We have called the effective entity that produces such compensation " $X$ " or cosmon and denoted with $\rho_{X}$ its energy density. Therefore, $\rho_{D}=\rho_{\Lambda}+\rho_{X}$ is the self-conserved total DE density in this context, which must be dealt with together with the ordinary density of matter $\rho_{M}$. A generic model of this kind is what we have called the $\Lambda \mathrm{XCDM}$ model [17,23].

Furthermore, from general considerations based on the covariance of the effective action of QFT in curved spacetime $[13,15,16]$, we expect that the running $\mathrm{CC}$ density $\rho_{\Lambda}=\rho_{\Lambda}(H)$ should be an affine quadratic law of the expansion rate $H$, see Eq. (67). Using this guiding principle and the ansatz of self-conservation of the DE, we find that the evolution of $\rho_{X}$, and hence of $\rho_{D}$, becomes completely determined, even though its ultimate nature remains unknown. In particular, $X$ is not a scalar field in general.

The $\Lambda \mathrm{XCDM}$ model was first studied in [17] as a promising solution to the cosmic coincidence problem, in the sense that the coincidence ratio $r=\rho_{D} / \rho_{M}$ can stay relatively constant, meaning that it does not vary in more than 1 order of magnitude for many Hubble times. The main aim of the present paper was to make a further step to consolidate such a possible solution of the coincidence problem, specifically from the analysis of the coupled system of matter and DE perturbations. Let us remark that this has been a rather nontrivial test for the $\Lambda$ XCDM model. Indeed, after intersecting the region where the DE perturbations of this model can be consistently defined, with the region where the coincidence problem can be solved $[17,23]$, we end up with a significantly more reduced domain of parameter space where the model can exist in full compatibility with all known cosmological data. The main conclusion of this study is that the predictivity of the model has substantially increased. Therefore, it can be better put to the test in the next generation of precision cosmological observations, which include the promising DES, SNAP, and PLANCK projects [63].

Interestingly enough, we have found that the final region of the parameter space is a naturalness region which is more accessible to the aforementioned precision experi- ments. For example, we have obtained the bound $0 \leqslant \nu \lesssim$ $\nu_{0} \sim 10^{-2}$ for the parameter that determines the running of the cosmological term. This bound is perfectly compatible with the physical interpretation of $\nu$ from its definition (68). Moreover, our analysis indicates that the cosmon entity $X$ behaves as "phantom matter" [17], i.e. it satisfies $\omega_{X}<-1$ with negative energy density. This result is a clear symptom (actually an expected one) from its effective nature. It is also a welcome feature; let us recall [17] that phantom matter, in contrast to the "standard" phantom energy, prevents the Universe from reaching the big rip singularity. Finally, perhaps the most noticeable (and experimentally accessible) feature that we have uncovered from the analysis of the DE perturbations in the $\Lambda \mathrm{XCDM}$ model, is that the overall EOS parameter $w_{e}$ associated to the total DE density $\rho_{D}$ behaves effectively as quintessence $\left(w_{e} \geq-1\right)$ in precisely the region of parameter space where the cosmic coincidence problem can be solved. In other words, quintessence is mimicked by the $\Lambda \mathrm{XCDM}$ model in that relevant region, despite the fact that there is no fundamental quintessence field in the present framework. A detailed confrontation of the various predictions of the $\Lambda \mathrm{XCDM}$ model [in particular, the kind of dependence $\left.w_{e}=w_{e}(z)\right]$ with the future accurate experimental data [63], may eventually reveal these features and even allow to distinguish this model from alternative DE proposals based on fundamental quintessence fields.

To summarize: we have demonstrated that the set of cosmological models characterized by a composite, and covariantly conserved, DE density $\rho_{D}$ in which the vacuum energy $\rho_{\Lambda}$ is a dynamical component [specifically, one that evolves quadratically with the expansion rate, see Eq. (67)], proves to be a distinguished class of models that may provide a consistent explanation of why $\rho_{D}$ is near $\rho_{M}$, in full compatibility with the theory of cosmological perturbations and the rest of the cosmological data. Remarkably, such a class of models is suggested by the above-mentioned renormalization group approach to cosmology. We conclude that the $\Lambda$ XCDM model can be looked upon as a rather predictive framework that may offer a robust, and theoretically motivated, dynamical solution to the cosmic coincidence problem.

\section{ACKNOWLEDGMENTS}

The authors are grateful to Julio Fabris for useful discussions. We have been supported in part by MEC and FEDER under Project No. FPA2007-66665 and also by DURSI Generalitat de Catalunya under Project No. 2005SGR00564. The work of J.G. is also financed by MEC under BES-2005-7803. We acknowledge the support from the Spanish Consolider-Ingenio 2010 program CPAN CSD2007-00042. 
[1] R. Knop et al., Astrophys. J. 598, 102 (2003); A. Riess et al., Astrophys. J. 607, 665 (2004).

[2] D. N. Spergel et al. (WMAP Collaboration), Astrophys. J. Suppl. Ser. 170, 377 (2007).

[3] N. N. Weinberg and M. Kamionkowski, Mon. Not. R. Astron. Soc. 341, 251 (2003); E. V. Linder, Phys. Rev. D 70, 043534 (2004).

[4] S. Cole et al., Mon. Not. R. Astron. Soc. 362, 505 (2005).

[5] O. Lahav et al., Mon. Not. R. Astron. Soc. 333, 961 (2002); L. Verde et al., Mon. Not. R. Astron. Soc. 335, 432 (2002).

[6] A. D. Dolgov, in: The Very Early Universe, edited by G. Gibbons, S. W. Hawking, and S. T. Tiklos (Cambridge University Press, Cambridge, England, 1982); L.H. Ford, Phys. Rev. D 35, 2339 (1987); J. Solà, Phys. Lett. B 228, 317 (1989).

[7] R. D. Peccei, J. Solà, and C. Wetterich, Phys. Lett. B 195, 183 (1987).

[8] C. Wetterich, Nucl. Phys. B302, 668 (1988); P. J.E. Peebles and B. Ratra, Astrophys. J. 325, L17 (1988); B. Ratra and P. J. E. Peebles, Phys. Rev. D 37, 3406 (1988); C. Wetterich, Astron. Astrophys. 301, 321 (1995); R. R. Caldwell, R. Dave, and P. J. Steinhardt, Phys. Rev. Lett. 80, 1582 (1998); For a review, see e.g. P. J. E. Peebles and B. Ratra, Rev. Mod. Phys. 75, 559 (2003), and the long list of references therein.

[9] S. Weinberg, Rev. Mod. Phys. 61, 1 (1989).

[10] See e.g. V. Sahni and A. Starobinsky, Int. J. Mod. Phys. D 9, 373 (2000); S. M. Carroll, Living Rev. Relativity 4, 1 (2001); T. Padmanabhan, Phys. Rep. 380, 235 (2003); E. J. Copeland, M. Sami, and S. Tsujikawa, Int. J. Mod. Phys. D 15, 1753 (2006); T. Padmanabhan, Gen. Relativ. Gravit. 40, 529 (2008).

[11] J. Solà and H. Štefančić, Phys. Lett. B 624, 147 (2005); Mod. Phys. Lett. A 21, 479 (2006); J. Phys. A 39, 6753 (2006).

[12] I. L. Shapiro and J. Solà, Phys. Lett. B 475, 236 (2000).

[13] I. L. Shapiro and J. Solà, J. High Energy Phys. 02 (2002) 006.

[14] A. Babic, B. Guberina, R. Horvat, and H. Štefančić, Phys. Rev. D 65, 085002 (2002); Phys. Rev. D 71, 124041 (2005).

[15] I. L. Shapiro and J. Solà, J. Phys. A 40, 6583 (2007).

[16] I. L. Shapiro and J. Solà, arXiv:hep-th/0808.0315.

[17] J. Grande, J. Solà, and H. Štefančić, J. Cosmol. Astropart. Phys. 08 (2006) 011; Phys. Lett. B 645, 235 (2007); J. Phys. A 40, 6787 (2007); J. Phys. A 40, 6935 (2007).

[18] I. L. Shapiro, J. Solà, and H. Štefančić, J. Cosmol. Astropart. Phys. 01 (2005) 012.

[19] J. Solà, J. Phys. A 41, 164066 (2008).

[20] L. Samushia and B. Ratra, Astrophys. J. 680, L1 (2008); arXiv:0810.2104; E. Gaztanaga, R. Miquel, and E. Sanchez, arXiv:0808.1921.

[21] W. J. Percival et al., Mon. Not. R. Astron. Soc. 381, 1053 (2007); S. Nesseris and L. Perivolaropoulos, J. Cosmol. Astropart. Phys. 01 (2007) 018.

[22] J. Q. Xia, H. Li, G. B. Zhao, and X. Zhang, Phys. Rev. D 78, 083524 (2008).

[23] J. Grande, R. Opher, A. Pelinson, and J. Solà, J. Cosmol. Astropart. Phys. 12 (2007) 007.
[24] E. V. Linder and A. Jenkins, Mon. Not. R. Astron. Soc. 346, 573 (2003).

[25] R. Opher and A. Pelinson, arXiv:astro-ph/0703779.

[26] P. J. Steinhardt, in: Critical Problems in Physics, edited by V.L. Fitch, D. R. Marlow, and M.A.E. Dementi (Princeton University, Princeton, NJ, 1997); P.J. Steinhardt, Phil. Trans. R. Soc. A 361, 2497 (2003).

[27] P. G. Ferreira and M. Joyce, Phys. Rev. D 58, 023503 (1998); P. J. Steinhardt, L. M. Wang, and I. Zlatev, Phys. Rev. D 59, 123504 (1999); V. Sahni and L. M. Wang, Phys. Rev. D 62, 103517 (2000).

[28] L. Amendola, Phys. Rev. D 62, 043511 (2000); L. Amendola and D. Tocchini-Valentini, Phys. Rev. D 64, 043509 (2001); W. Zimdahl and D. Pavon, Phys. Lett. B 521, 133 (2001).

[29] S. Dodelson, M. Kaplinghat, and E. Stewart, Phys. Rev. Lett. 85, 5276 (2000); S. Nojiri and S. D. Odintsov, Phys. Lett. B 637, 139 (2006).

[30] T. Chiba, T. Okabe, and M. Yamaguchi, Phys. Rev. D 62, 023511 (2000); C. Armendariz-Picon, V.F. Mukhanov, and P. J. Steinhardt, Phys. Rev. D 63, 103510 (2001).

[31] R. R. Caldwell, Phys. Lett. B 545, 23 (2002); R. R. Caldwell, M. Kamionkowski, and N. N. Weinberg, Phys. Rev. Lett. 91, 071301 (2003); A. Melchiorri, L. Mersini, C. J. Odman, and M. Trodden, Phys. Rev. D 68, 043509 (2003); H. Štefančić, Phys. Lett. B 586, 5 (2004); S. Nojiri and S. D. Odintsov, Phys. Rev. D 70, 103522 (2004); R. J. Scherrer, Phys. Rev. D 71, 063519 (2005); S. Capozziello, S. Nojiri, and S.D. Odintsov, Phys. Lett. B 632, 597 (2006).

[32] S. Deser and R. P. Woodard, Phys. Rev. Lett. 99, 111301 (2007).

[33] A. Y. Kamenshchik, U. Moschella, and V. Pasquier, Phys. Lett. B 511, 265 (2001); M. C. Bento, O. Bertolami, and A. A. Sen, Phys. Rev. D 66, 043507 (2002); J. C. Fabris, S. V. B. Goncalves, and P.E. de Souza, Gen. Relativ. Gravit. 34, 53 (2002).

[34] S. Weinberg, Phys. Rev. Lett. 59, 2607 (1987); H. Martel, P. R. Shapiro, and S. Weinberg, Astrophys. J. 492, 29 (1998); A. Vilenkin, Phys. Rev. D 50, 2581 (1994), and references therein.

[35] P.J.E. Peebles, Physical Principles of Cosmology (Princeton University, Princeton, NJ, 1993); T. Padmanabhan, Structure Formation in the Universe (Cambridge University Press, Cambridge, England, 1993); A.R. Liddle and D.H. Lyth, Cosmological Inflation and Large Scale Structure (Cambridge University Press, Cambridge, England, 2000); V.F. Mukhanov, Physical Foundations of Cosmology (Cambridge University Press, Cambridge, England, 2005).

[36] V.F. Mukhanov, H. A. Feldman, and R. H. Brandenberg, Phys. Rep. 215, 203 (1992).

[37] K. A. Malik, D. Wands, and C. Ungarelli, Phys. Rev. D 67, 063516 (2003); R. Bean and O. Doré, Phys. Rev. D 69, 083503 (2004).

[38] J. M. Bardeen, Phys. Rev. D 22, 1882 (1980).

[39] H. Kodama and M. Sasaki, Prog. Theor. Phys. Suppl. 78, 1 (1984). See version with corrections in [40].

[40] T. Hamazaki and H. Kodama, Prog. Theor. Phys. 96, 1123 (1996). 
[41] J. C. Fabris, I. L. Shapiro, and J. Solà, J. Cosmol. Astropart. Phys. 02 (2007) 016.

[42] J. K. Erickson, R. R. Caldwell, P. J. Steinhardt, C. Armendariz-Picon, and V.F. Mukhanov, Phys. Rev. Lett. 88, 121301 (2002).

[43] W. Hu, Astrophys. J. 506, 485 (1998).

[44] T. Koivisto and D. F. Mota, Phys. Rev. D 73, 083502 (2006).

[45] A. Vikman, Phys. Rev. D 71, 023515 (2005).

[46] R. R. Caldwell and M. Doran, Phys. Rev. D 72, 043527 (2005).

[47] W. Hu, Phys. Rev. D 71, 047301 (2005); B. Feng, X. L. Wang, and X. M. Zhang, Phys. Lett. B 607, 35 (2005); Z. K. Guo, Y. S. Piao, X. M. Zhang, and Y.Z. Zhang, Phys. Lett. B 608, 177 (2005); L. Perivoralopoulos, Phys. Rev. D 71, 063503 (2005).

[48] M. Takada, Phys. Rev. D 74, 043505 2006; S. Unnikrishnan, H. K. Jassal, and T.R. Seshadri, arXiv: astro-ph/0801.2017.

[49] I. L. Shapiro, J. Solà, C. España-Bonet, and P. RuizLapuente, Phys. Lett. B 574, 149 (2003); C. EspañaBonet, P. Ruiz-Lapuente, I. L. Shapiro, and J. Solà, J. Cosmol. Astropart. Phys. 02 (2004) 006; I. L. Shapiro and J. Solà, Nucl. Phys. B, Proc. Suppl. 127, 71 (2004); Report No. AHEP2003/013, 2004.

[50] R. Foot, A. Kobakhidze, K. L. McDonald, and R. R. Volkas, Phys. Lett. B 664, 199 (2008).
[51] L.S. Brown, Quantum Field Theory (Cambridge University Press, Cambridge, England, 1994).

[52] R. Horvat, Phys. Lett. B 648, 374 (2007).

[53] S. Dodelson, Modern Cosmology (Academic, New York, 2003).

[54] J. M. Bardeen, J. R. Bond, N. Kaiser, and A. S. Szalay, Astrophys. J. 304, 15 (1986).

[55] N. Sugiyama, Astrophys. J. Suppl. Ser. 100, 281 (1995).

[56] S. M. Carroll, W. H. Press, and E. L. Turner, Annu. Rev. Astron. Astrophys. 30, 499 (1992).

[57] C. L. Bennett et al., Astrophys. J. 464, L1 (1996).

[58] R. Stompor, K. M. Gorski, and A. J. Banday, Mon. Not. R. Astron. Soc. 277, 1225 (1995).

[59] G. Hinshaw et al., Astrophys. J. Suppl. Ser. 170, 288 (2007).

[60] L. R. Abramo, R. C. Batista, L. Liberato, and R. Rosenfeld, J. Cosmol. Astropart. Phys. 11 (2007) 012; arXiv:0806.3461.

[61] H. A. Borges, S. Carneiro, J. C. Fabris, and C. Pigozzo, Phys. Rev. D 77, 043513 (2008); Phys. Rev. D 78, 123522 (2008).

[62] J. He, B. Wang, and E. Abdalla, Phys. Lett. B 671, 139 (2009).

[63] http://www.darkenergysurvey.org/; http://snap.lbl.gov/; http://www.rssd.esa.int/index.php?project=planck. 